\title{
Evaluation of the National Adherence \\ Guidelines for Chronic Diseases in South Africa
}

\section{HEALTHCARE PROVIDER PERSPECTIVES ON DIFFERENTIATED CARE MODELS}

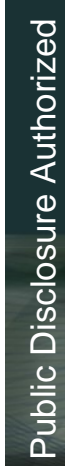

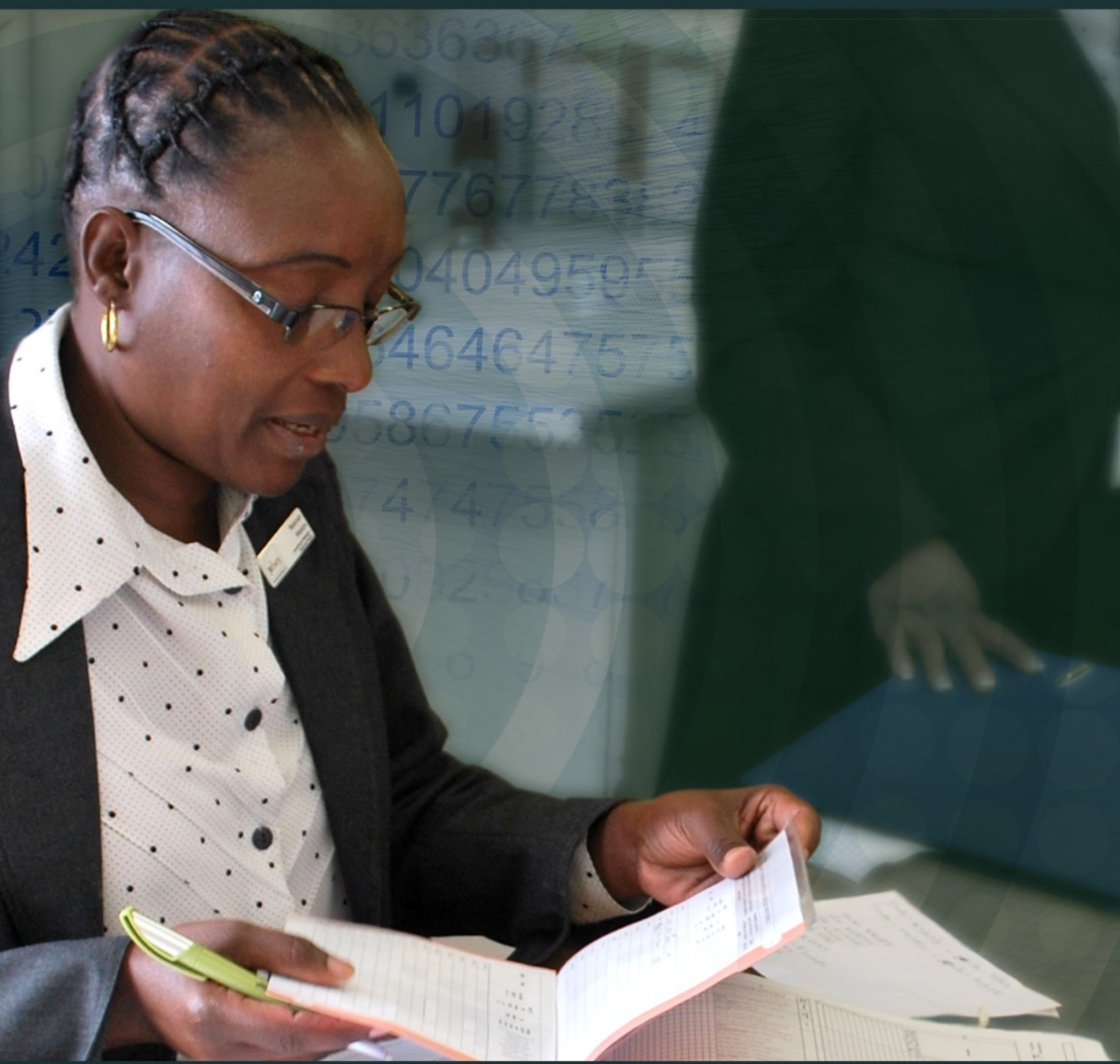

JUNE 2017

NW UKaid
BOSTON UNIVERSITY
HERO

$H E^{2} R O$ 
(C) International Bank for Reconstruction and Development / The World Bank 1818 H Street NW, Washington DC 20433

Internet: www.worldbank.org; Telephone: 2024731000

This work is a product of The World Bank and its contractor vendor, Boston University. The findings, interpretations, and conclusions expressed in this work do not necessarily reflect the views of the Executive Directors of The World Bank or other partner institutions or the governments they represent. The World Bank does not guarantee the accuracy of the data included in this work. The boundaries, colors, denominations, and other information shown on any map in this work do not imply any judgment on the part of The World Bank concerning the legal status of any territory or the endorsement or acceptance of such boundaries.

\section{Rights and Permissions}

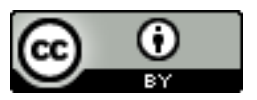

This work is available under the Creative Commons Attribution 3.0 Unported licence (CC BY 3.0) http://creativecommons.org/licences/by/3.0. Under the Creative Commons Attribution license, you are free to copy, distribute and adapt this work, including for commercial purposes, under the following conditions:

Attribution - Please cite the work as follows: Evaluation of the National Adherence Guidelines for Chronic Diseases in South Africa: Healthcare Provider Perspectives on Different Care Models, 2017. Washington DC: World Bank. License: Creative Commons Attribution CC BY 3.0

Translations - If you create a translation of this work, please add the following disclaimer along with the attribution: This translation was not created by The World Bank and should not be considered an official World Bank translation. The World Bank shall not be liable for any content or error in its translation.

All queries on rights and licenses should be addressed to the Office of the Publisher, The World Bank, $1818 \mathrm{H}$ Street NW, Washington DC, 20433, USA; fax: 202-522-2625; email: pubrights@worldbank.org. 


\section{Evaluation of the \\ National Adherence Guidelines for Chronic Diseases in South Africa}

\section{HEALTHCARE PROVIDER PERSPECTIVES ON DIFFERENTIATED CARE MODELS}

JUNE 2017

World Bank co-authors: Nicole Fraser, Zara Shubber, Marelize Görgens 
This page is for collation purposes 


\section{TABLE OF CONTENTS}

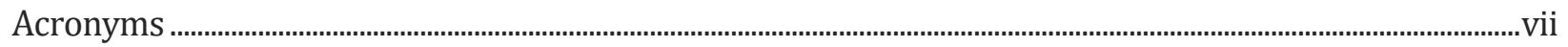

Acknowledgements ................................................................................................................................................................ ix

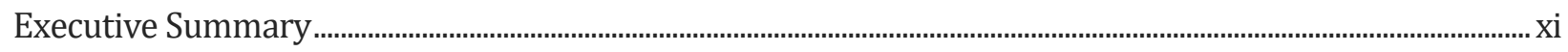

ANSWERS TO THE STUDY QUESTIONS ……….......................................................................................... xii

KEY MESSAGES AND RECOMMENDATIONS .............................................................................................. xiv

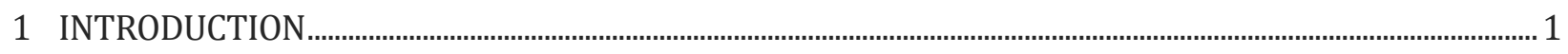

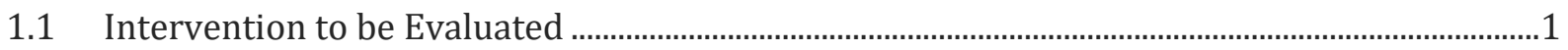

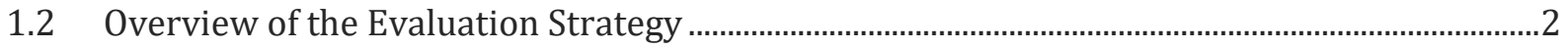

1.3 Implementation Evaluation Aim ......................................................................................................

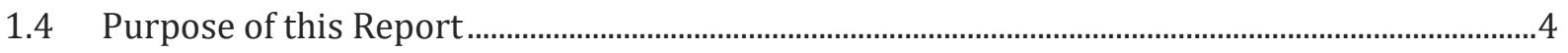

2 IMPLEMENTATION EVALUATION DESIGN AND METHODS …….......................................................................... 5

2.1 Implementation Evaluation Questions …………...............................................................................

2.2 Implementation Evaluation Framework....................................................................................

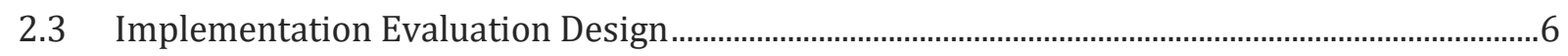

2.4 Implementation Evaluation Data Collection Methods...................................................................

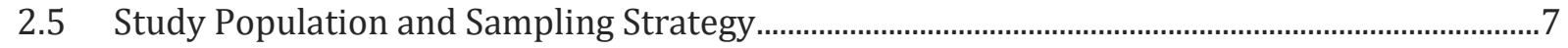

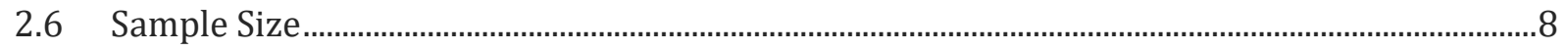

2.7 Data Analysis and Interpretation ..................................................................................................

3 FINDINGS..................................................................................................................................

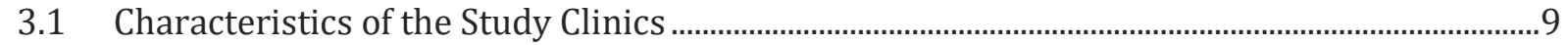

3.2 Characteristics of the Provider Respondents............................................................................. 10

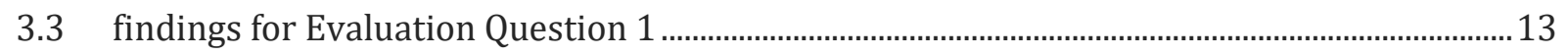

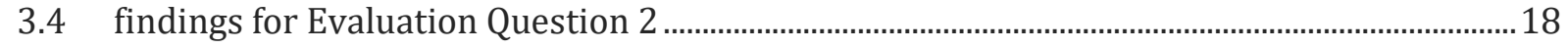

3.5 findings for Evaluation Question 3 and 4................................................................................... 36

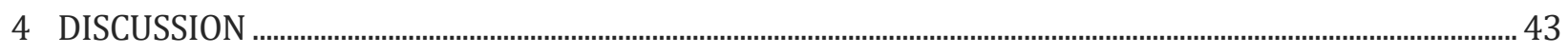

4.1 KEY MESSAGES AND RECOMMENDATIONS …………............................................................. 45

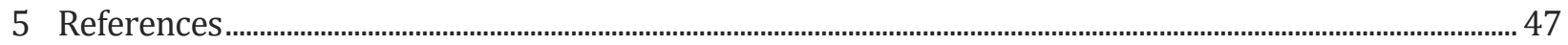




\section{APPENDIX}

Appendix 1 - Project Logic Model 40

\section{FIGURES}

1.1 Theoretical framework to assess program success through intervention and implementation effectiveness

\section{TABLES}

1.1 National Adherence Guidelines minimum package of interventions ................................................ 1

2.1 Themes for the evaluation of the Implementation of the Minimum Package under the Consolidated Framework for Implementation Research (CFIR).......................................................... 6

3.1 Matched pairs included in the process evaluation, site allocation and Decentralized Medication Delivery (DMD) implementation .........................................................................................10

3.2 Health Care Provider cadres interviewed as part of the process evaluation and role in implementing AGL interventions

3.3 General Characteristics of Provider In-depth Interview Respondents for the 48 included provider interviews.

3.4 Most frequently cited perceived barriers and facilitators to implementation of the minimum package interventions among interviewed providers by intervention and control arm

3.5 AGL interventions and corresponding activities in the care cascade by type of patient supported.

3.6 Most frequently cited themes of perceived strengths and weaknesses of testing and initiation activities among interviewed providers by intervention and control arm.

3.7 Most frequently cited cross-cutting suggestions for adherence strategies among provider IDI respondents by intervention and control groups

3.8 Most frequently cited suggestions for adherence strategies among interviewed provider by intervention and control groups.. 


\title{
ACRONYMS
}

\author{
AC Adherence Club \\ AGL Adherence Guidelines \\ ART Antiretroviral Therapy \\ CCMDD Central Chronic Medication Distribution and Delivery \\ CDU Central Dispensing Unit \\ CFIR Consolidated Framework for Implementation Research \\ DMD Decentralized Medication Delivery \\ EAC Enhanced Adherence Counselling \\ ETR Electronic TB Register \\ FTIC Fast Track Initiation Counselling \\ HIV Human Immunodeficiency Virus \\ IDI In-depth Interviews \\ NCD Non-communicable Disease \\ NDOH National Department of Health \\ NGO Non-Governmental Organizations \\ OM Operational/Facility Manager \\ PN Professional Nurse \\ RPCS Repeat Prescription Strategy \\ TB Tuberculosis \\ TRIC Tracing and Retention in Care \\ SFLA Spaced Fast-Lane Appointments \\ VL Viral Load \\ WBOT Ward Based Outreach Team
}


This page is for collation purposes 


\section{ACKNOWLEDGEMENTS}

This report is part of the impact evaluation led by the South African National Department of Health, in collaboration with the NDOH's National Health Laboratory Service, World Bank, Boston University and the University of Witwatersrand. The impact evaluation has three Principal Investigators: Mokgadi Phokojoe (National Department of Health), Nicole Fraser (World Bank) and Matthew Fox (Boston University). The evaluation was funded by the Government of South Africa, the World Bank and the UK Department for International Development.

The following individuals are part of the evaluation team and contributed to this report:

National Department of Health: Mokgadi Phokojoe (Director: Care and Support), Tshepo Malapo (M\&E Manager), Lillian Diseko (Deputy Director for HIV Care and Treatment) and Yogan Pillay (Deputy Director-General for Health)

World Bank: Nicole Fraser, Zara Shubber, Marelize Görgens

Boston University: Matthew Fox, Sydney Rosen, Nancy Scott and Rachel Fong University of Witwatersrand (Health Economics and Epidemiology Research Office (HE2RO)): Sophie Pascoe, Amy Huber, Joshua Murphy and Aneesa Moolla

The South Africa National Department of Health and the rest of the research team would like to thank all the health care managers and providers in the study provinces, districts and facilities for their participation and support in this evaluation.

All graphics in the report were designed by Theo Hawkins, product designer and Knowledge Management Officer at the World Bank HIV team 
This page is for collation purposes 


\section{EXECUTIVE SUMMARY}

In June of 2016, a team of researchers from South Africa's National Department of Health, the World Bank, Boston University and the Health Economics and Epidemiology Research Office began enrolling patients into an evaluation of the National Department of Health's National Adherence Guidelines (AGL) for Chronic Diseases being implemented by the National Department of Health (NDoH). The AGL is a tool for the NDoH to promote differentiated care, a client-centred approach that simplifies and adapts services across the HIV cascade while reducing unnecessary burdens on the health system.

The AGL evaluation determines both effectiveness and implementation learning, using five patient cohorts and guided by two protocols. The five cohorts are meant to evaluate five interventions within the AGL to improve adherence to HIV care: Fast track initiation counselling (FTIC), decentralized medication delivery (DMD), adherence clubs (AC), early patient tracing (TRIC) and enhanced adherence counselling (EAC). The evaluation is designed as a cluster randomized evaluation design in 24 sites (12 intervention and 12 control in 4 provinces). Protocol 2

This report presents the result of the qualitative evaluation to understand the implementation of five interventions from the provider perspective.

(implementation learning) sampled providers from eight sites.

This report presents the result of the qualitative evaluation to understand the implementation of five interventions from the provider perspective. We conducted in-depth interviews with 48 providers at eight sites, one intervention and one control site in each of the four provinces. The report sought to answer four key questions:

1. What are the barriers to and facilitators of implementing the minimum package interventions from the perspective of the providers?

2. What are the strengths and weaknesses of each intervention for HIV positive patients from the perspective of providers?

3. How could implementation of adherence interventions and the minimum package of interventions be improved?

4. What additional strategies do providers feel would be helpful in improving treatment adherence?

For each question we systematically coded and used thematic analysis of the qualitative interview transcripts, to understand emerging themes. We illustrated emergent themes with direct, illustrative quotes from intervention and control respondents. 


\section{ANSWERS TO THE STUDY QUESTIONS}

What are the barriers to and facilitators of implementing the minimum package interventions from the perspective of the providers?

- A lack of human resources was the primary barrier to implementing adherence interventions at intervention sites.

- Pre-packed medication parcels not being delivered by the time the patient was scheduled for pickup was the primary barrier to implementation of the CCMDD model of DMD.

What are the strengths and weaknesses of each intervention for HIV positive patients from the perspective of providers?

Interventions for HIV treatment initiation:

- Providers from intervention sites articulated only strengths and were generally supportive of the FTIC model

- Providers from control sites spoke more frequently about test and treat, but expressed reluctance about treating without waiting for laboratory results

- The counselling component of FTIC was favourably received at intervention sites and seen as an important part of the FTIC strategy; this will likely be key to generating support for the approach when implemented at control sites

\section{Interventions for patients stable on HIV treatment and adherent:}

- Stable patients had several options for enrolling into a repeat prescription strategy and providers saw this as being a positive addition to the clinics

- Adherence clubs were generally viewed positively by providers as they allowed patients to meet in groups and give support to each other with a potential increase in adherence

- DMD was also seen as a good way to decongest clinics, though it was noted that when it was poorly implemented and patients could not receive their medications when scheduled to do so, it created problems for patients and providers

\section{Interventions for patients failing treatment or who missed visits:}

- Providers saw enhanced adherence counselling as an important strategy for improving adherence for those with an elevated viral load. However, they noted that EAC brought an additional burden to the clinic in terms of provider time and could not always be delivered if not enough staff were available

- Providers also saw tracing as an important strategy for getting patients to return to care, but there was a strong feeling that they had limited resources to be able to complete tracing activities and were not always welcomed in the community to trace patients 
How could implementation of adherence interventions and the minimum package of interventions be improved?

- Providers generally suggested that in order to improve the interventions, more resources are needed, in particular more staff time

- Providers expressed a need for stronger initial training as well as ongoing mentorship and training

- Providers recommended that all sites be fully equipped with supplies, materials and trained providers

\section{What additional strategies do providers feel would be helpful in improving treatment adherence?}

- Leveraging the links between community health workers and communities and involving community leaders was seen by providers as a key way to improve uptake of interventions

- Providers also recommended conducting campaigns and using the media (such as radio) to notify communities about health care changes and the services available

The results show that providers were generally positive about all the interventions, though they had mixed comments about DMD and TRIC, largely because they were not always well implemented or providers felt they did not have the resources to implement them well. Additionally, providers' views were mixed on their perceived effectiveness of AC. On the one hand, clubs were seen as a place for patients to share adherence experiences while also greatly shortening the time they needed to spend in the clinic. On the other hand, many patients simply wanted to collect their medication and depart quickly. Providers also noted that it was difficult to staff adherence clubs with all the other activities they had to implement, and some sites phased them out in favour of DMD. Interestingly, providers noted that the repeat prescription strategies were seen as providing benefits not just to the patient enrolled in them, but also to more complicated patients who remained at the clinic as providers could spend more time with them. The repeat prescription strategies also alleviated congestion in the clinics, but the challenge of medicines not being delivered in time for DMD (often referred to as "stock outs") emerged as an issue to be addressed. Providers generally suggested that in order to improve the interventions, more resources are needed, in particular more staff time.

As we enter into the long term follow up phase of the evaluation, As we enter into the long term follow up phase of the evaluation, we will be able to use the results of these provider interviews to provide context to the results of the effectiveness evaluation and use this information to seek ways to improve the rollout of the adherence interventions. we will be able to use the results of these provider interviews to provide context to the results of the effectiveness evaluation and use this information to seek ways to improve the rollout of the adherence interventions. 


\section{KEY MESSAGES AND RECOMMENDATIONS}

- The potential for the AGL to reduce the burden on clinic staff is recognised but only if there are sufficient HR resources to implement the guidelines. At scale-up it is therefore important to ensure that HR staff shortages are addressed particularly for non-clinical cadres such as lay counsellors, data capturers and community health workers.

- The issue of readiness of sites for implementation (i.e. materials, supplies, training, etc.) needs to be addressed before scale up so that providers feel empowered to implement effectively and have the support of their district managers.

- It is important to engage with staff to identify the appropriate people for training; adequate initial training and ongoing mentorship of staff are also key to ensuring effective implementation of the AGL.

- Targets and priorities for decongestion of patients and test and treat need to be clearly communicated in the context of implementing the AGL and appropriate training for all strategies is required.

- Issues around DMD implementation need to be resolved, adequate systems need to be put in place to ensure that data are fed back to the facilities and the ability to get the medications to the pickup points at the scheduled times must be addressed.

- One of the key strengths of the repeat prescription collection strategies is the element of patient choice (adherence clubs, pick-up points outside the facility, spaced fast lane appointments at the facility). The appropriate resources should be kept in place to ensure this element of patient choice remains.

- While the counselling component of FTIC and EAC was favourably received, the introduction of adherence plans and completion of these during counselling has caused confusion and a perception of being burdensome. Continued use of these plans needs to be carefully considered and, if continued, detailed training on their completion and accessing replacement books is required.

- The need for the type of supporting materials developed for the AGL was highlighted but these materials may need to be more readily available for facilities and all cadres of clinic staff as national scale up of the AGL continues.

- Links with communities and community leaders should be leveraged to promote adherence and treatment services and media sources should be utilised to inform patients about changes to health care provision and the introduction of new services. 


\section{INTRODUCTION}

For antiretroviral therapy (ART) for HIV and treatment for other chronic diseases to be effective, patients must remain in care for long periods of time, initiate treatment as early as allowed under prevailing guidelines, consistently achieve high levels of adherence to their treatment regimen and, as a result, exhibit stable monitoring test results and/or treatment completion. In the case of HIV, treatment is lifelong and requires consistent, nearly complete adherence to sustain an undetectable viral load. Numerous studies and reviews (1-3) as well as the South African National Department of Health's (NDOH) own data, have indicated that retention in care and adherence to ART in South Africa are sub-optimal and pose a serious threat to the long-term success of the national HIV response.

\subsection{INTERVENTION TO BE EVALUATED}

To address the retention and adherence to HIV challenge, in 2014 the South Africa NDOH developed the "National Adherence Guidelines for Chronic Diseases (HIV, TB and NCDs)". The guidelines address the provision of a minimum package of interventions to increase linkage to care, retention in care, and adherence to treatment. They reflect a differentiated care approach which aims to enhance the quality of the patient's experience as well as reduce the burden on the health system so that health system resources can be reallocated to those patients most in need. The minimum package interventions are listed in Table 1.1, five of which are being evaluated under this study, with a focus on HIV.

Table 1.1 National Adherence Guidelines minimum package of interventions

\begin{tabular}{ll}
\hline APPROACH & INTERVENTION \\
\hline Education and counselling & $\begin{array}{l}\text { Fast track initiation counseling* } \\
\text { Enhanced adherence counseling for unstable patients* } \\
\text { Child disclosure counseling for children living with HIV }\end{array}$ \\
\hline $\begin{array}{l}\text { Repeat prescription collection } \\
\text { strategies }\end{array}$ & $\begin{array}{l}\text { Adherence clubs* } \\
\text { Spaced and fast lane appointment systems } \\
\text { Decentralised medication delivery* }\end{array}$ \\
\hline Patient tracing & Early tracing of all missed appointments* \\
\hline Integrated HIV, TB, NCD care & Integrated consultation and counselling \\
\hline Note: $*$ Indicates interventions included in this evaluation \\
Source: Authors
\end{tabular}


Before launching nationwide scale-up of the Adherence Guidelines ${ }^{1}$ (AGL), the NDOH has selected 12 clinics (primary health care clinics and community health centres) for early implementation of the minimum package for HIV patients. These sites were intended to generate information to refine the guidelines, gain experience in implementation, and project budgetary needs. The early implementation sites were also intended to generate data on the effectiveness of the interventions and on how to best tailor and target the. These early-learning sites, therefore, provided a platform upon which to build a rigorous evaluation strategy.

\subsection{OVERVIEW OF THE EVALUATION STRATEGY}

Evaluation of the AGL has been designed as a mixed methods matched cluster-randomised evaluation with 12 early learning (intervention) sites implementing the minimum package of interventions during the early-learning phase of national roll-out, and 12 delayed implementation (control) sites which delayed most aspects of implementation of the minimum package interventions until the national roll-out. All implementation of the interventions is being conducted by NDOH in South Africa.

The effectiveness of interventions, once taken to scale, is highly dependent on the effectiveness of the implementation, typically grounded in organizational behaviour and conformity theory. The field of implementation sciences is evolving rapidly as researchers and policy makers recognize the need to understand not only how well the intervention works, but also how to best deliver or implement the intervention at scale. Figure 1.1 illustrates the importance of understanding both the effectiveness of interventions as well as the effectiveness of implementation of those interventions to ensure overall program effectiveness. This theoretical framework guides our approach of evaluating the AGL.

The effectiveness of interventions, once taken to scale, is highly dependent on the effectiveness of the implementation, typically grounded in organizational behaviour and conformity theory.

The evaluation strategy includes two parallel and complementary studies. First, we are estimating the effectiveness of the AGL on patient outcomes in a study entitled "Evaluation of the National Department of Health's National Adherence Guidelines for Chronic Diseases in South Africa Using Routinely Collected Data" (Approved by Boston University IRB BUIRB H-34074 and University of Witwatersrand Human Research Ethics Committee Wits Protocol M150537). This effectiveness evaluation (shown the lower panel of Figure 1) is a matched, cluster-randomized design in 24 clinics, 12 of which received early implementation of the minimum package and 12 of which delayed implementation and serve as control sites. Prior to being randomly allocated to intervention and control, clinics were matched into pairs on four clinic characteristics: total

1 National Department of Health, South Africa. Adherence Guidelines for HIV, TB and NCDs. https://www.nacosa.org.za/wpcontent/uploads/2016/11/Integrated-Adherence-Guidelines-NDOH.pdf. Guidelines Standard Operating Procedures found at http://www.differentiatedcare.org/Portals/0/adam/Content/_YiT3_-

qmECUkmkpQvZAIA/File/SOP\%20A5\%20booklet\%2020-05-2016.pdf 
remaining on ART, clinic size, setting, location and viral suppression. This study uses routinely collected data at sites to understand how the minimum package affects patient outcomes.

Second, we seek to understand the implementation process in a study entitled "Process Evaluation of the National Department of Health's Adherence Guidelines for Chronic Diseases in South Africa" (BUIRB H34197 and Wits Protocol M150652). Using data not routinely collected from a selection of sites within the cluster randomized design, we can assess why the interventions worked or did not work, for whom they worked, and what conditions are associated with greater or lesser effectiveness. This "implementation evaluation" (shown in the upper panel of Figure 1.1) uses data collected from patients and providers to understand the implementation process, intervention acceptability, patient and provider preferences and views of the intervention.The individual adherence interventions included in the minimum package require different implementation activities and produce different outputs and outcomes in the patient population, but they seek to converge to improve adherence and health outcomes in the larger patient population (Appendix 1, program logic model). Therefore, findings from the intervention effectiveness evaluation and the implementation evaluation can be triangulated to understand overall programmatic effectiveness of the National AGL and generate recommendations for improvement as the NDOH continues to roll out the AGL.

Figure 1.1 Theoretical framework to assess program success through intervention and implementation effectiveness

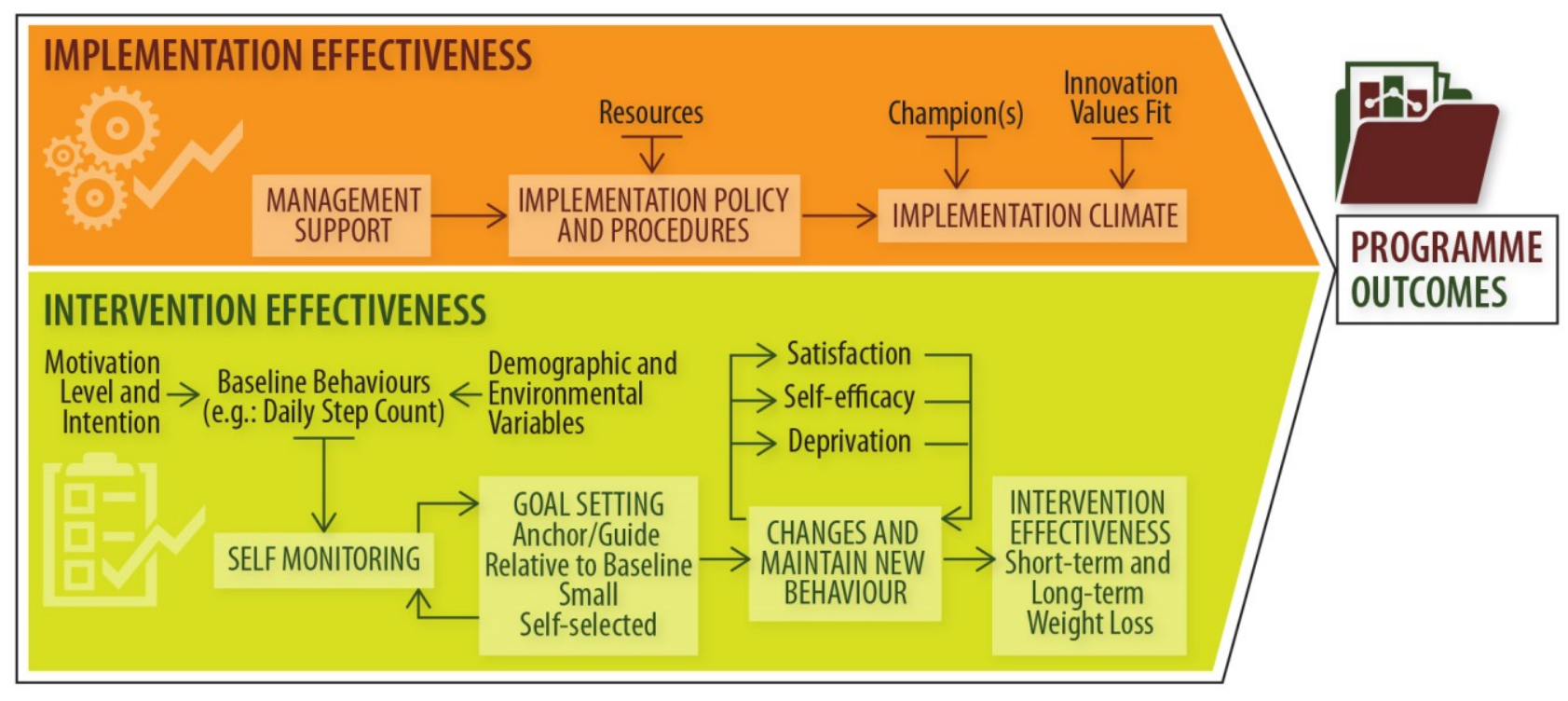

Source: Damschroder L (2012) The role and selection of theoretical frameworks in implementation research.

\subsection{IMPLEMENTATION EVALUATION AIM}

The overall aim of this component of the evaluation is to understand key aspects of the implementation of the AGL from the perspective of the patients and providers and, in the longer term, to explore how these components relate to the effectiveness of each minimum package intervention on patient outcomes. The evaluation will describe and assess which interventions are actively taken up by the evaluation sites, the processes by which the interventions in the minimum 
package are being implemented, patients' acceptance of the interventions, patients' perceptions of the quality of care associated with the interventions, and providers' perspectives on the experience of implementing the interventions at the site.

The objectives of this implementation evaluation are:

1. to assess the degree to which each individual intervention in the minimum package was correctly implemented in accordance with national guidelines

2. to understand the implementation strategy at each clinic and to compare the types of strategies used between intervention and control sites to improve treatment adherence

3. to assess the impact of each of the minimum package interventions on perceived quality of care and identify factors that influence patients' acceptance of each intervention and assess the impact of perceived quality of care on treatment outcomes

4. to assess factors that influence acceptability of each intervention to providers

\subsection{PURPOSE OF THIS REPORT}

The purpose of this report is to describe the perspectives of facility providers at both intervention and control sites on implementing the minimum package of interventions. Findings presented here are intended to inform the $\mathrm{NDOH}$ as it continues to plan for national rollout of the minimum package. Lessons learned from this evaluation are intended to help NDOH improve targeting of resources, staff training, communications materials, data systems, and other critical elements of the implementation of the AGL. 


\section{IMPLEMENTATION EVALUATION DESIGN AND METHODS}

\subsection{IMPLEMENTATION EVALUATION QUESTIONS}

The four evaluation questions answered in this report include:

1. What are the barriers to and facilitators to implementing the minimum package interventions from the perspective of the providers?

2. What are the strengths and weaknesses of each intervention for HIV positive patients from the perspective of providers?

3. How could implementation of adherence interventions and the minimum package of interventions be improved?

4. What additional strategies do providers feel would be helpful in improving treatment adherence?

\subsection{IMPLEMENTATION EVALUATION FRAMEWORK}

To answer the evaluation questions, we used a mixed-methods approach, guided by the Consolidated Framework for Implementation Research (CFIR) (Table 2.1). CFIR broadly assesses the key implementation domains of: 1) intervention characteristics (quality, adaptability, complexity); 2) outer setting (patient needs, setting, policies); 3) inner setting (organizational priority, implementation climate, leadership engagement); 4) individual provider characteristics (knowledge about interventions, self-efficacy, identification with organization) and; 5) process (planning, engaging, executing and evaluating). This report focuses on the in-depth interviews (IDI) of the Provider Themes. 
Table 2.1 Themes for the evaluation of the Implementation of the Minimum Package under the Consolidated Framework for Implementation Research (CFIR)

\begin{tabular}{|c|c|}
\hline CFIR DOMAIN & CFIR CONSTRUCTS RELEVANT TO THE IDI PROVIDER THEMES \\
\hline 1. Intervention characteristics & $\begin{array}{l}\text { - Intervention source } \\
\text { - Evidence strength and quality } \\
\text { - Relative advantage } \\
\text { - Complexity } \\
\text { - Adaptability } \\
\text { - Design quality and packaging }\end{array}$ \\
\hline 2. Outer setting & $\begin{array}{l}\text { - Patient needs and resources } \\
\text { - External policies }\end{array}$ \\
\hline 3. Inner setting & $\begin{array}{l}\text { - Organizational structure and support } \\
\text { - Implementation climate } \\
\text { - Relative priority } \\
\text { - Barriers and facilitators to implementation } \\
\text { - Impact on organization }\end{array}$ \\
\hline 4. Individual provider characteristics & $\begin{array}{l}\text { - Knowledge and beliefs about interventions } \\
\text { - Self-efficacy } \\
\text { - Impact on provider } \\
\text { - Other provider attributes }\end{array}$ \\
\hline 5. Process & $\begin{array}{l}\text { - Implementation planning } \\
\text { - Engagement } \\
\text { - Implementation leaders and champions } \\
\text { - Execution } \\
\text { - Reflecting and evaluating }\end{array}$ \\
\hline
\end{tabular}

Source: Damschroder LJ, Aron DC, Keith RE, Kirsh SR, Alexander JA, Lowery JC: Fostering implementation of health services research findings into practice: A consolidated framework for advancing implementation science

\subsection{IMPLEMENTATION EVALUATION DESIGN}

Embedded within the effectiveness evaluation, the implementation evaluation used a crosssectional design and a mixed-methods approach to collect data from a sub-sample of providers at four intervention and four control sites. This approach has several strengths. First, it allows for triangulating findings from various data sources. Second, the control group allows us to differentiate the challenges posed by implementing the minimum package interventions from those that are already faced by clinics. Third, because the implementation evaluation is embedded within the effectiveness evaluation, at the end of the study we will be able to relate implementation data to patient outcome data and observe changes over time. 


\subsection{IMPLEMENTATION EVALUATION DATA COLLECTION METHODS}

Data were collected using in-depth interviews (IDI) among providers over a period of six months, from November 2016 through April 2017. The development of the semi-structured, qualitative IDI guides was informed by the WHO quality of care domains (health care effective, efficient, accessible, acceptable/patient-centred, equitable and safe). IDI's were conducted with different cadres of healthcare providers at each site in order to gain a deeper understanding of their experience implementing adherence strategies.

\subsection{STUDY POPULATION AND SAMPLING STRATEGY}

Provider interviews were conducted at a subsample of eight of the 24 effectiveness evaluation sites. We purposively selected with $\mathrm{NDOH}$ one pair of intervention and control sites from each of the four provinces for a total of eight sites.

Potential participants for the provider interviews were identified by purposively selecting one provider from each appropriate cadre of provider and inviting them to participate. At intervention sites we specifically sought to invite those providers responsible for implementing the interventions. At control sites we identified those providers who were involved in the implementation of adherence strategies as part of the standard of care at those sites or who would be responsible for implementing the AGL interventions The development of the semi-structured, qualitative IDI guides was informed by the WHO quality of care domains (health care effective, efficient, accessible, acceptable/patientcentred, equitable and safe). when rolled out at those sites. Where there were several staff members of the same cadre eligible we picked the member of staff who was available to be interviewed and in all cases included the Clinic/Operations Manager. We purposively identified a maximum of six respondents at each of the four intervention sites and four control sites, with the intention of stopping earlier if we reached saturation or predictability.

The provider IDIs included direct providers of each of the interventions (e.g., lay counselors, nurses, pharmacists) at intervention sites as well as clinic operations managers and non-clinical staff indirectly involved in implementation (e.g., data capturers, administrative clerks).

\subsubsection{Inclusion and Exclusion Criteria}

Providers were eligible to participate in the IDIs if they:

- Played some role in implementing adherence strategies or approaches at the facility.

- Were currently working at one of the study health facilities as a provider.

- $\geq 18$ years old

Providers were excluded from participating in the IDIs if they:

- Were unwilling to provide informed consent 


\subsubsection{Data Collection, Management and Storage}

A researcher certified in human subjects protection and trained in qualitative methods administered the interviews at the clinics at a time convenient for the clinic staff member. Before the interview, the researcher conducted an informed consent process and documented participant consent with a signature on an approved informed consent form. Names and ID numbers were not recorded in order to reduce the risk of breaches of confidentiality. Each interview lasted 30-60 minutes. IDIs were audio recorded, translated and transcribed verbatim. Paper copies of qualitative transcripts and notes were kept in a locked cabinet. Transcripts are kept in password-protected files and all identifying information has been removed from the qualitative datasets.

\subsection{SAMPLE SIZE}

We estimated interviewing a maximum of 48 providers, sufficient to be practical and to reach saturation or predictability before reaching the maximum sample size.

\subsection{DATA ANALYSIS AND INTERPRETATION}

All qualitative data were analysed in NVivo 11॰ (Doncaster, Australia). Coding themes were identified $a$ priori according to the evaluation questions and the CFIR Framework. Additional themes were included as they emerged. Two researchers coded the Provider IDI transcripts and we assessed intercoder agreement by calculating a Kappa coefficient and refined the coding as necessary until agreement reached good correlation $(>0.5)$. We then analysed themes by intervention type and by intervention and control, per the evaluation question. 


\section{FINDINGS}

This section first presents a description of the sample, followed by the results for each of the evaluation questions.

\subsection{CHARACTERISTICS OF THE STUDY CLINICS}

Provider data were collected from 8 clinics, a sub-sample of four matched pairs from the overall impact evaluation study sample. One pair was selected in each district. Table 3.1 presents the 8 selected sites by district and province and the intervention allocation at these sites. As seen in Table 3.1, due to competing priorities at the National level and separate targets to decongest stable patients out of health facilities, DOH's Decentralized Medication Delivery (DMD) implementation happened outside the confines of randomisation. The result of this was DMD implementation at some control sites and absence of DMD at some intervention sites. To account for this, impact of the DMD intervention is being analysed as an observational study in the effectiveness evaluation and in the process evaluation we need to be cognizant of where DMD implementation has occurred (as noted in the last column of Table 3.1). Those sites that were implementing DMD at the time of cohort enrollment (effectiveness evaluation) are listed below as "DMD int at enrollment". However, it should also be noted that some sites were not implementing DMD at the time of enrollment for the effectiveness evaluation but have since started implementing DMD (indicated below as the DMD control site with an *). As such it is possible that even at DMD control sites, some providers may have noted that DMD is now being provided at their facility. In addition, it should also be noted that two different models of DMD were implemented at sites,

The model of DMD implementation needs to be considered as we review the successes and challenges of the DMD intervention. depending on district. In Ekurhuleni, the Chronic Dispensing Unit model is used where medications are packaged by the District Pharmacist and dispensed to facilities and pick up points. In the other three districts Central Chronic Medication Distribution and Delivery (CCMDD) uses a model in which medications are pre-packaged by private providers (e.g., Medipost, Pharmadirect, Optipharm) and distributed to approved pick-up-points including facilities. Even though CCMDD is at some sites delivered to facilities, for the purpose of this evaluation we focussed on those pick-up-points that were external to the facility to prevent confusion with the spaced fast lane appointment process for picking up medications at facilities. The model of DMD implementation needs to be considered as we review the successes and challenges of the DMD intervention. Furthermore, given the high targets that were set for DMD at the national level a further level of complexity is introduced at certain sites where they decided to take patients out of adherence club cohorts and move them to DMD instead (e.g., Tlhabane and Thokozani) resulting in additional complications for the evaluation at those intervention sites implementing both Adherence Clubs (AC) and DMD. 
Table 3.1 Matched pairs included in the process evaluation, site allocation and Decentralized Medication Delivery (DMD) implementation

\begin{tabular}{llll}
\hline PROVINCE & SITE NAME & SITE ALLOCATION & DMD IMPLEMENTATION \\
\hline Gauteng, Ekurhuleni SD S2 & Motsamai & Intervention & DMD int at enrollment \\
& Tamaho & Control & DMD int at enrollment \\
\hline Limpopo, Mopani & Tzaneen & Intervention & DMD Control \\
& Nkowankowa CHC & Control & DMD Control \\
\hline North West, Bojanala & Tlhabane & Intervention & DMD int at enrollment \\
& Bafokeng & Control & DMD int at enrollment \\
\hline KwaZulu Natal, uThungulu & King Dinizulu & Intervention & DMD int at enrollment
\end{tabular}

Notes: DMD int at enrollment: DMD intervention at time of enrollment; DMD Control: DMD control at time of enrollment; *=DMD control at time of enrollment but started implementing DMD after HIV cohort enrollment.

\subsection{CHARACTERISTICS OF THE PROVIDER RESPONDENTS}

We completed all of the projected 48 interviews. Table 3.2 below identifies all those health care provider cadres that were interviewed and the respective role that each cadre plays in implementation of the AGL. We interviewed 6 providers at each site selecting from the cadres below and purposively selecting those who were responsible for implementation of AGL interventions at intervention sites or for adherence approaches more generally at control sites. During the analysis, we reached saturation and predictability, key markers to cease qualitative analysis once 22 of the interviews had been coded although we continued coding transcripts to ensure no key themes were missed.

Table 3.2 Health Care Provider cadres interviewed as part of the process evaluation and role in implementing AGL interventions

HEALTH CARE ROLE IN IMPLEMENTING AGL INTERVENTIONS

PROVIDER

Operational/ Clinical provider responsible for all operational management at the facility including budget and Facility resource management. Responsible for oversight/supervision of AGL implementation at the facility, Manager ensuring that all appropriate cadres are trained, that resources including materials and support tools (OM) are available and for defining staff responsibilities for implementation; they may also be responsible for reviewing and signing of blood results before filing. 
Table 3.2 Health Care Provider cadres interviewed as part of the process evaluation and role in implementing AGL interventions (continued)

\section{HEALTH CARE ROLE IN IMPLEMENTING AGL INTERVENTIONS PROVIDER}

Professional Clinical provider responsible for: screening for chronic conditions; counselling (in absence of lay nurse (PN) counsellor) and testing for HIV; vitals; diagnosis of HIV and other chronic conditions (in absence of doctor); initiation of treatment; scripting of repeat chronic medications and distributing medications; health education; review of blood work and sign off of viral load results prior to filing (if required by the OM); and providing next appointment dates. At those sites implementing the AGL: a professional nurse often completes the final stage of Fast Track Initiation Counselling (FTIC) including completion of adherence plans and initiation of treatment; may assist with Enhanced Adherence Counselling (EAC) and identifying those patients eligible for EAC from viral load (VL) results; may be responsible for scripting meds for Repeat Prescription Strategy (RPCS); pre-packing medications for AC and responsible for oversight of and sometimes facilitation of ACs; also responsible for informing patients about tracing and obtaining patient consent for Tracing and Retention in Care (TRIC).

Enrolled nurse Clinical provider with a similar role to the professional nurse although not able to write prescriptions but can distribute pre-packaged medications prescribed by doctor or PN. May assist with facilitation of AC but plays a lesser role in implementation of the AGL.

Lay counsellor Non-clinical facility staff who under the AGL are responsible for pre- and post-test counselling of FTIC patients including completion of an adherence plan, may undertake counselling of EAC patients. They may also assist with the facilitation of ACs and phone tracing of TRIC and patients who are defaulting on treatment.

Pharmacist Clinical provider who under the AGL is responsible for pre-packing and dispensing medications for all FTIC and EAC patients and TRIC patients who return to care. They may also pre-pack medications for an AC (if that $\mathrm{AC}$ is not a DMD pick-up-point) They will also pre-pack and dispense medications for any patient allocated in spaced fast lane appointments (SFLA) and must document medication pick ups for these patients. In addition, they may also have oversight of all scripting processes for DMD ensuring that the appropriate paperwork is completed.

Pharmacists Clinical provider who plays the same role as the pharmacist particularly for the AGL interventions. At Assistant most facilities, there is rarely a full-time pharmacist but more often there is a pharmacist assistant either part or fulltime.

Community Outreach providers who may or may not be part of a Ward based outreach team (WBOT). They are health worker/ assigned to a facility and responsible for assisting with tracing of TRIC patients. This may include Ward Based phone tracing at the facility as well as household visits to all patients who have missed a visit or are Outreach Team defaulting on treatment. It may also include the tracing of patients with high viral loads. (WBOT) Leader

Health Non-clinical facility staff who conduct group health-talks which may include talks about adherence. In promoter terms of the AGL they may raise awareness about AGL interventions, particularly the repeat prescriptions collection strategies and if also in charge of queue marshalling may direct patients for counselling sessions at those sites.

Data capturer Non-clinical facility staff who do not as a rule have any direct-interaction with patients. They are responsible for capturing all HIV patient data recorded on the patient file on TIER and for other diseases on the relevant database (e.g. ETR for TB) where these exist at the facility. Depending on the facility data capturers can also be responsible for pulling patient files prior to visits and for filing them again after they have been captured. With the new TIER modules that are being introduced they will be responsible for capturing $A C$ and DMD register information that flows back to the facility. They are responsible for capturing viral loads and any back log can impact implementation of EAC and identification of patients eligible for RPCS. They may also assist with identifying patients who have missed visits and are therefore eligible for TRIC.

Source: Authors. 
As seen in Table 3.3 the majority of providers who were interviewed were female, particularly at intervention sites. This unequal gender breakdown is common in many South African facilities with male healthcare providers being far fewer in the primary healthcare setting. At the intervention sites around a quarter of those interviewed were non-clinical cadres of staff such as lay counsellors, health promoters and data capturers and an additional $10 \%$ were outreach staff such as community health workers and ward-based outreach team members (see Table 3.2). This was slightly higher at intervention sites which is to be expected given the role these cadres play in implementation of the minimum package of AGL interventions. At control sites where these interventions have yet to be implemented, more clinical providers (nurses and pharmacists) were interviewed indicating the role they currently play in supporting adherence approaches and interventions. The median number of years working at or with the facility was slightly longer in the intervention than the controls sites ( 4.0 years vs 3.0 years) as was the median number of years working with the facility in their current capacity (5.5 years at intervention sites vs 3.5 years at control sites).

Table 3.3 General Characteristics of Provider In-depth Interview Respondents for the 48 included provider interviews

\begin{tabular}{|c|c|c|c|c|c|c|}
\hline \multirow[b]{2}{*}{ Characteristic } & \multicolumn{2}{|c|}{ INTERVENTION (N=24) } & \multicolumn{2}{|c|}{ CONTROL (N=24) } & \multicolumn{2}{|c|}{ TOTAL (N=48) } \\
\hline & $n$ & (\%) & $n$ & (\%) & $n$ & (\%) \\
\hline \multicolumn{7}{|l|}{ Gender } \\
\hline Female & 22 & $92 \%$ & 17 & $71 \%$ & 39 & $81 \%$ \\
\hline Male & 2 & $8 \%$ & 7 & $29 \%$ & 9 & $19 \%$ \\
\hline \multicolumn{7}{|l|}{ Current Role } \\
\hline Clinical Provider & 15 & $63 \%$ & 16 & $67 \%$ & 31 & $65 \%$ \\
\hline Non-clinical facility staff & 7 & $29 \%$ & 5 & $21 \%$ & 12 & $25 \%$ \\
\hline Outreach & 2 & $8 \%$ & 3 & $13 \%$ & 5 & $10 \%$ \\
\hline $\begin{array}{l}\text { Median \# years working with } \\
\text { facility/organization (range) }\end{array}$ & \multicolumn{2}{|c|}{$4.0(1.0,16.0)$} & \multicolumn{2}{|c|}{$3.0(1.0,19.0)$} & \multicolumn{2}{|c|}{$3(1.0,19.0)$} \\
\hline $\begin{array}{l}\text { Median \# years working with } \\
\text { in current capacity (range) }\end{array}$ & \multicolumn{2}{|c|}{$5.5(0.0,21.0)$} & \multicolumn{2}{|c|}{$3.5(1.0,26.0)$} & \multicolumn{2}{|c|}{$4.5(0.0,26)$} \\
\hline
\end{tabular}

Source: Authors.

\subsubsection{Discussion}

One matched pair of intervention and control sites were selected from each of the four AGL early learning districts, to attempt to ensure that data would capture the implementation experience commonalities and differences across areas. No new themes emerged, after the initial 22 transcripts were coded, but additional quotes that reinforce existing themes were identified and incorporated, validating that saturation had been reached. The respondents worked in different cadres of the health system, and therefore represent a variety of perspectives. There were sufficient respondents to understand experiences implementing each of the interventions, or, in the case of controls, the general areas in which each intervention could be implemented. 


\subsubsection{Key Messages}

i. 4 matched pairs (intervention and control) were selected representing one intervention and one control site from each of the four districts

ii. Respondents are from different cadres of health providers who can reflect on experiences implementing the minimum package from control and intervention sites

iii. The sample analyzed was sufficient to reach saturation giving us confidence that no additional themes will emerge

\subsection{FINDINGS FOR EVALUATION QUESTION 1}

What are the barriers and facilitators to implementing the minimum package interventions from the perspective of the providers?

\subsubsection{Barriers to implementation}

As seen below in Table 3.4, the most frequently cited barriers to implementing the minimum package interventions from the perspective of the providers at both intervention and control sites included clinic level challenges and a general lack of resources.

Table 3.4 Most frequently cited perceived barriers and facilitators to implementation of the minimum package interventions among interviewed providers by intervention and control arm

\begin{tabular}{|c|c|c|c|}
\hline \multicolumn{2}{|c|}{ INTERVENTION (N=10) } & \multicolumn{2}{|c|}{ CONTROL $(\mathrm{N}=12)$} \\
\hline 3arriers & Facilitators & Barriers & Facilitators \\
\hline $\begin{array}{l}\text { Clinic level barriers } \\
\text { including a shortage of } \\
\text { staff, and fatigued staff } \\
\text { Safety of staff when } \\
\text { conducting tracing } \\
\text { Stigma within the } \\
\text { community or perceived } \\
\text { at the health facility } \\
\text { /Distance to facility to } \\
\text { access care }\end{array}$ & $\begin{array}{l}\text { - Clinic level facilitators } \\
\text { including district } \\
\text { implementation } \\
\text { support and a strong } \\
\text { team } \\
\text { - Available resources } \\
\text { including medication } \\
\text { and air time }\end{array}$ & $\begin{array}{l}\text { - Lack of resources including } \\
\text { medication arriving late or } \\
\text { not at all, limited money, } \\
\text { airtime for communication } \\
\text { and pamphlets } \\
\text { - Clinic level barriers including } \\
\text { insufficient staff, untrained } \\
\text { staff } \\
\text { - Forms were complicated } \\
\text { and staff had limited time to } \\
\text { complete the forms }\end{array}$ & $\begin{array}{l}\text { - Available resources } \\
\text { including community health } \\
\text { workers and NGOs } \\
\text { - Lack of stigma/No queues } \\
\text { - Clinic level facilitators } \\
\text { including adequate training } \\
\text { and personnel }\end{array}$ \\
\hline
\end{tabular}

Source: Authors 
Clinic level barriers for the intervention group primarily included a shortage of staff at the clinic resulting in patients having to wait to see those healthcare providers who were delivering the interventions. Providers expressed this lack of a sufficient number of providers as leading to fatigue among staff and an inability to take on additional interventions:

"Maybe, if our government can think for us. Like, for instance, here, with me here. I'm a 24-hour service clinic, only operating with eight professional nurses. Others are on night shift, and out of those, they are working day shift. So, it's very difficult. With the huge number of those 22,000 and above patients. Eight professional nurses. So, that is why our nurses, you see, it seems as if, even though they do get the information they're not able to implement it. Because they are tired.

Yes." Clinical Provider, Intervention site

Intervention arm respondents also noted concerns with implementing outreach into the community, such as when tracking patients who missed appointments as part of TRIC, as barriers to implementation of the interventions. Long distances needed to be travelled and safety issues for the staff and outreach workers were identified as barriers to implementation:

"And there were boys in a group, group of boys. They were calling us names like, hey girls, come here. Then we decided that it's not safe and we didn't even finish registration on that side because of that. We were not safe". Outreach Staff, Intervention Site

Physical safety concerns emerged, to a lesser degree, in the control staff as well.

"The plan is maybe only if we can talk to the tracers and go deep into its space. So, as I was saying earlier on, where we're getting, where we are tracing it's not a safe community, so maybe sometimes they are scared, sometimes, you never know, but for my site, it's not a safe area for the girls only to go there."

Non-clinical facility staff, Control site

Clinic level barriers that emerged from the control group primarily included a lack of adequate resources such as medication, money, airtime, and educational materials or pamphlets. These are resources that are essential for implementing the minimum package of interventions, with airtime being a well noted barrier to implementing TRIC (or standard of care tracing) at both intervention and control sites:

"The issue which I said for communication. They must also use their own airtime, if they don't have airtime it means they won't implement."

Clinical Provider, Control Site (referencing tracing interventions)

Most frequently, respondents in the control arm mentioned medication parcels not arriving on time as barriers to implementing their interventions, and this was especially the case in control sites 
which implemented DMD and more specifically CCMDD (this was most pronounced at Bafokeng and Nkwalini and was less of an issue at Tamaho where the Central Dispensing Unit (CDU) DMD model was implemented ). The providers noted that DMD was not always well implemented, leading to confusion for patients and for providers. The problem often related to a disconnect between the timing of delivery of medications and the times at which patients were asked to collect their medications:

"The fact that they're not delivering the patients' parcels on time. That happens very often. Like, for this whole week, there have been patients that have been trying to collect their medication; medication wasn't here. And then, yesterday, they probably delivered. I wasn't here yesterday, my colleague was. They delivered, and then this... because this morning, when I go there, I saw there was a whole bunch of boxes that were delivered, but some of those dates had already passed. You understand?... What I always tell the patients is if they come collect their medication and their medication isn't here, I can't guarantee that they're going to get it. Sorry" Clinical Provider, Control Site - DMD intervention at enrollment

Control site respondents also found the forms, particularly for those implementing CCMDD, were complicated and they did not feel sufficiently trained:

"But out of the CCMDD, it's only the forms that were given. Then forms that were not, they were not user-friendly, especially for return dates of the patients. It was confusing. And then they edited it and then they made other forms together with the cards, the cards that should be given to patients who were enrolled." Clinical Provider, Control site

Additionally, as illustrated by the quotes below, control arm respondents perceive a shortage of human resources as a barrier to handling the large volume of patients at the clinics:

"... because we are faced with [unclear] situation where by we are grossly and severely short staffed most of the time. So it's difficult for us in our existing numbers to deal with the huge population of people coming to the clinic still with patients."

Facility Management, Control Site - DMD Control*

"Now it won't be possible because there's no staff, because it needs manpower and we don't have manpower."

Clinical Provider, Control Group 


\subsubsection{Facilitators to implementation}

As Table 3.4 above indicates, the most frequently cited facilitators for implementation of adherence interventions among intervention arm respondents included clinic level facilitators, namely strong team members, and a general availability of resources.

"Yes, this rotation has helped us, because if I'm not here, and if the next person doesn't know what is happening, so then what will happen to the community? So it just helps to rotate." Clinical Provider, Intervention Site

Above, rotation appears to refer to staff redundancy where more than one person is trained to do a job so they can rotate and fill in for one another. Below, respondents illustrate the support they feel having a strong team and sufficient support by community health workers:

"The working relationship between me and my colleagues. I think that ... because we should be able to work together, then we should be able to work efficiently, and then work will progress

$$
\text { efficiently." }
$$

Clinical Provider, Intervention Site

Additionally, intervention respondents felt supported by their district staff and noted this as a facilitator to effectively implementing the adherence interventions.

"As [the District staff] come, we think they are very supportive because even when we have challenges, we have to tell them that we have challenges about this and that. That's why I say, they are supportive."

Non-clinical facility staff, Intervention Site

Facilitators of implementation of adherence interventions most frequently indicated by the control site respondents were the assistance of community health workers, and implementation support by partner non-governmental organizations. They identified this cadre of staff as essential for promoting adherence and noted that they play a role that allows the clinic based staff to focus on their tasks at the facility, freeing them to work with the patients who present at the sites:

"The thing that made it easier is only the community healthcare workers who go out there and look for those patients and receive those patients. Because ... as professionals, we can't go and receive them. It's time consuming. You can't go there after your work. If you go there, you have to make it during your work time and then when we go there, who's going to stay there and help those who are in the clinic? So the community healthcare workers, they make it very easy because they are the ones who go and receive those patients."

Clinical Provider, Control Site 
Control site respondents at sites implementing DMD also noted that trainings or workshops, and the provision of standard operating procedures, were helpful with DMD implementation (particularly CCMDD implementation) as illustrated by the following responses:

"I will say, because [trainers] were available all the time to assist in everything maybe that was needed by us, it was much easier for implementation because in most cases, [trainers] were available at the facility." Clinical Provider, Control Site implementing DMD

In regards to training being seen as a facilitator of implementation of the DMD intervention, it was also emphasized by a respondent that training the right and an adequate number of personnel plays an important role in implementing both CCMDD and the CDU model of DMD. This is illustrated below:

“..the assistant that works here that's responsible for dispensing the medication and helping the patients find their medication, they weren't exactly part of the training. So, the pharmacist in charge of the health centre went for the training, but she's not the one here every day...seeing the patients and dealing with CCMDD. So, there was training ... I don't think that it goes to all the people that it needed to get to." Clinical Provider, Control site - DMD intervention at enrollment

\subsubsection{Discussion on barriers and facilitators to implementing the minimum package interventions}

While both intervention and control respondents cited a lack of human resources as a barrier to implementation, it was more pronounced among intervention site respondents. This is likely because they are currently implementing the AGL interventions and allowed them to highlight human resource challenges related to the specific interventions in the minimum package (e.g., sufficient lay counsellors to do fast-track initiation counselling and community outreach workers to do tracing). Even though control site respondents are not specifically implementing the guideline interventions, human resources still present a barrier to implementing standard of care adherence approaches (e.g., enhanced adherence counselling, fast-track initiation of priority patients and tracing). However, we found that control sites that were implementing the CCMDD model of DMD were more likely to identify barriers related to implementing this intervention, such as drugs not being available at pick up points on time.

Overall, the intervention group respondents noted that motivated staff and a supportive implementation environment was helpful, or facilitated, the implementation of the AGL. Notably, absence of medications did not emerge as a theme among the intervention respondents potentially because of variations in how successfully DMD was implemented at different sites. During the evaluation period some sites started CCMDD, then later stopped, while in others, patients were shifted between AC and DMD (both CDU and CCMDD). In these sites, medication pickups were noted as challenging for some patients if medications were not prepared for patients at the time 
they arrived for their scheduled pickup. The issues mentioned around patients not being able to pick up their medication in DMD do not appear to be related to medications in general, but rather to medications at CCMDD pick up points in cases where medication were not delivered on time or there was confusion with when patients should pick up their medications or because there had been issues with the scripting process for DMD.

The barriers mentioned at the control sites show some important insights around implementing the minimum package. While the control sites were not implementing the overall AGL, most of them were fast-tracking initiation of treatment, at least for some patient types, and therefore were also conducting adherence counselling prior to initiation. In addition, many control sites were providing enhanced adherence counselling for patients with high viral loads as these strategies are outlined under the current HIV treatment guidelines. Some level of tracing, particularly of patients who missed appointments by more than two weeks or who had high viral loads were also occurring at control sites. In KwaZulu Natal some control sites had also previously implemented adherence clubs and models of DMD whereby the facilities had pre-packed medications to deliver to specified pick-up-points. Respondents in

Respondents in the control group primarily said that implementing adherence interventions or strategies was facilitated by trainings and workshops, as well as support from community health workers. the control group primarily said that implementing adherence interventions or strategies was facilitated by trainings and workshops, as well as support from community health workers.

\subsubsection{Key Messages}

i. A lack of human resources was the primary barrier to implementing adherence interventions at intervention sites.

ii. Pre-packed medication parcels not being delivered by the time the patient was scheduled for pickup was the primary barrier to implementation of the CCMDD model of DMD.

\subsection{FINDINGS FOR EVALUATION QUESTION 2}

What are the strengths and weakness of each intervention for HIV positive patients from the perspective of the providers?

We asked respondents from intervention and control sites to comment on the strengths and weaknesses of the activities for adherence support that are undertaken throughout the cascade of treatment. We did not ask specifically about the AGL interventions for several reasons. First, not all respondents use the same terminology when describing the AGL interventions. Second, as part of standard qualitative practice we did not want to ask leading questions. Third, we aimed to compare the experiences of the intervention and control respondents, and therefore needed to ask the same 
questions around common themes. The general themes (described in Table 3.5 below) are mapped to corresponding AGL interventions.

Table 3.5 AGL interventions and corresponding activities in the care cascade by type of patient supported

\begin{tabular}{|c|c|c|}
\hline TYPE OF PATIENT & ACTIVITIES ON CASCADE OF CARE & CORRESPONDING AGL INTERVENTION \\
\hline \multirow{5}{*}{$\begin{array}{l}\text { New patients testing and } \\
\text { initiating treatment }\end{array}$} & Testing & Fast Track Initiation Counselling (FTIC) \\
\hline & Initiation on treatment & Adherence Plan \\
\hline & Counselling around time of & \\
\hline & initiation & \\
\hline & Lab testing & \\
\hline \multirow[t]{3}{*}{ Patients stable and adherent } & Routine collection of medication & Adherence Clubs (AC) \\
\hline & Support groups & Decentralized medicine delivery (DMD) \\
\hline & & Spaced fast-lane appointments (SFLA) \\
\hline \multirow{4}{*}{$\begin{array}{l}\text { Patients who are unstable on } \\
\text { treatment (either failing } \\
\text { treatment or non-adherent) or } \\
\text { at risk of being lost from care }\end{array}$} & Appointment cards & Enhanced adherence counselling (EAC) \\
\hline & Outreach & Tracing and retention in care (TRIC) \\
\hline & Home visits & \\
\hline & Missed visit tracking & \\
\hline
\end{tabular}

Source: Authors.

Below we present the results by the type of patient the respondents were commenting on (e.g., patients initiating treatment, patients stable on treatment, patients unstable on treatment, patients lost to care).

\subsubsection{Results: Patient testing and initiation of treatment}

Table 3.6 illustrates the most common themes that emerged among respondents when discussing the strengths and weaknesses of patient testing and initiation on treatment. The strengths of Fast Track Initiation Counselling (FTIC) emerged quite clearly, with most responses being around faster initiation of treatment such that patients do not have to return later or wait for CD4 results. We note that this will likely change as South Africa's Universal HIV Test and Treat policy no longer requires patients to wait for a $\mathrm{CD} 4$ count result to initiate treatment.

Table 3.6 Most frequently cited themes of perceived strengths and weaknesses of testing and initiation activities among interviewed providers by intervention and control arm

\begin{tabular}{|c|c|c|c|}
\hline \multicolumn{2}{|c|}{ INTERVENTION (N=10) } & \multicolumn{2}{|c|}{ CONTROL (N=12) } \\
\hline Strengths & Weaknesses & Strengths & Weaknesses \\
\hline $\begin{array}{l}\text { - Counseling } \\
\text { - Initiation of treatment } \\
\text { - Testing quickly }\end{array}$ & $\begin{array}{l}\text { - Stress of developing } \\
\text { adherence plan }\end{array}$ & $\begin{array}{l}\text { - Reluctance around rapid } \\
\text { initiation of treatment } \\
\text { - Testing }\end{array}$ & $\begin{array}{l}\text { - Initiation of treatment } \\
\text { - Testing }\end{array}$ \\
\hline
\end{tabular}

Sources: Authors.

The analysis revealed that no themes of weakness around testing and initiation activities emerged among the intervention respondents in the AGL arm. The most frequent strengths intervention respondents identified were around the benefits of initiating treatment quickly, such that early treatment would be beneficial for patients' health as illustrated below: 
"Okay, it's helped them because it's avoid other diseases like TB. They... when they take treatment early, like normally when he starts treatment with HIV they normally give them other treatment to avoid TB for six months."

Outreach staff, Intervention site

Additionally, providers noted that the counselling component of fast track initiation was important for the success of the intervention. They noted that one of the benefits of initiating HIV treatment rapidly was keeping patients in care and completing the steps necessary to initiate antiretroviral therapy. However they also noted that it was important to ensure the patient understood the reasons why they should start treatment quickly particularly in light of the current role out of 'treatment for all':

"At first I think we had to be trained how are we going to make the patient to understand why do we have to do all these things. Like, doing initiation at the same day, because, some of the patients understand that we have to come to the clinic and do the testing, you take some bloods and go home and come back the other day...But now, you have to prepare them how to cope with this. And to make sure they understand why do they have to do this, to do it at the same day. Because some of the patients, if you say go home and come back another day. Some of the patients don't come. And they will tell you, I had no transport."

Non-Clinical Facility Staff, Intervention Site

"So that they will know how to take their medication, when to take their medication and also to know the side effects and to report back if there is anything wrong."

Clinical provider, Intervention site

We note that the first quote mentions same day treatment initiation which is not specifically part of FTIC where initiation occurs alongside a second counselling session, not the first.

The only weakness intervention respondents identified around FTIC was the additional burden of having to complete an adherence plan for each patient. They noted that they found filling in the

"I think it is the adherence plan. The adherence plan was not here before, right. So, now if you do the class, you have to fill in this patient adherence plan. Which we, I think sometimes, is very stressful. You have to make sure that every patient, you have to complete this adherence plan." Non-Clinical Facility Staff, Intervention site

patient's adherence plan to be stressful when being completed for so many patients:

While in general providers had positive things to say about FTIC, among some respondents there appeared to be some confusion around the difference between FTIC and test and treat as the two were implemented at roughly the same time and with similar goals of getting patients onto treatment earlier and faster. 
"Before they normally, before they will take bloods and then you will find out maybe the CD4 count is above five-hundred. They won't start treatment. But now with this FTIC, fast track, now they start treatment immediately."

Outreach, Intervention site

"We begin by counselling them so that they are able to accept their statuses. These days we no longer wait for the CD4 count." Non-Clinical Facility Staff, Intervention site

Control site respondents were motivated by the benefits of testing and initiation of treatment regardless of FTIC, and were likely responding more to the test and treat guidelines as illustrated below:

"I get very excited when I see a patient being tested for HIV and then that patient comes back for the results. You know you'll be giving that patient maybe three days to come for the result, that patient will come. And then you take that patient for adherence counselling before we start the treatment. That patient will go and then start the treatment. Maybe that patient will be, like, not well, acutely ill or chronically ill when he comes first. So, after two weeks when the patient comes back, you see a very different person. So, that thing, it makes me to want to give more, to want to see more patients who can be on ART and get help."

Clinical provider, Control site

"Okay. Alright, fast track initiation well presently we are ensuring that we do test as many people as possible. Because in the fight of HIV and in the aim of eradicating HIV pandemic by 2020 we need to test as many people as possible. $90 \%$ of the population that is HIV positive have to know, they have to know their HIV status. So, we test as many people as possible and in our health talks education every morning we do ask people to come and test. And indeed, they do test. In September, I know this when testing was introduced we tested around 500 to 600 people. And amongst those we initiated around 63 patients if I'm not mistaken."

Clinical Provider, Control site

Control site respondents were concerned with the notion of initiating patients immediately and concerned that they required blood work prior to initiation, though again, this likely is also related to the test and treat policy that was implemented at the same time as the AGL were being implemented. 
"And so we are continuing doing so. For patients who are tested HIV positive on the same day we do take the baseline. Because I declined completely to adopt this style of initiating patients same day. Because there are some other factors that could not warrant that. You know I mean the TB the [unclear] you never know about all that if you just initiate the patient the same day. Besides if you do initiate the patient the same day you may not be encouraged or be urged to take the baseline bloods. And because you wouldn't see a significance of taking that. Whereas it's very important to take baseline bloods because with baseline bloods and baseline information and baseline investigations we can detect things like TB. We cannot start ARVs on the patient if they have TB you have to deal with it first. Then after around eight weeks after the patient is stable and able to stand it you can initiate the patient and start on the ARVs." Clinical Provider, Control site

This confusion between test and treat and fast tracking initiation as well as well as fast-tracking picking up medication was further echoed by the providers who on occasion seemed unclear about the different interventions and eligibility outlined in the AGL.

"Everyone is happy. But the concern was that when the first time they said about CCMDD, they said they must be fast tracking something. Like said you will test and treat. Which doesn't make sense, because we have to take bloods, and then we treat regarding to the blood." Clinical provider, Control site - DMD Control*

\subsubsection{Discussion: Patient testing and initiation of treatment}

Respondents from both intervention and control sites spoke to the rapid initiation of treatment, likely in response to the new HIV treatment guidelines that were recently implemented determining that treatment should now be offered to all those who are HIV positive irrespective of their CD4 count at diagnosis. Those implementing FTIC spoke to the benefits of rapid initiation and, more salient to FTIC, the counselling component. Most of the benefits noted from the intervention providers were focused on the perceived benefits to the patient such as better management or prevention of co-morbidities, and helping the patients cope with their illness and treatment burden as well as preventing pretreatment loss to follow up. The themes that emerged strongly suggested that the counselling aspect of FTIC was one of its biggest strengths as it gave providers a tool to help patients understand the responsibilities of taking treatment.

Notably, the control site respondents who were not implementing

The themes that emerged strongly suggested that the counselling aspect of FTIC was one of its biggest strengths as it gave providers a tool to help patients understand the responsibilities of taking treatment.

FTIC did not discuss the benefits of counselling at all. Control site respondents primarily discussed the removal of CD4 thresholds and seemed to confuse test and treat with fast tracking. In practice, control sites would tend to fast track priority patients only (i.e., pregnant, low CD4, etc.) and in 
general, expressed that it was more beneficial for patients to wait for test results, rather than initiate immediately.

\subsubsection{Key Messages: Testing and initiation of treatment}

i. Providers from intervention sites articulated only strengths and were generally supportive of FTIC, particularly around faster treatment initiation

ii. Providers from control sites spoke more frequently about test and treat, but expressed reluctance about treating without waiting for labs

iii. The counselling component of FTIC was favourably received at intervention sites and seen as an important part of the FTIC strategy. This will likely be key to generating support for the approach when implemented at control sites

\subsubsection{Results: Patients stable and adherent}

AGL interventions for patients who are stable and adherent focus primarily on repeat prescription medicine collection strategies: Adherence Clubs (AC) and Decentralized medicine delivery (DMD).

\section{Adherence Clubs}

Intervention site respondents found that adherence clubs were popular among their patients and that patients learned about them by word of mouth with other patients recommending them based on their positive experiences. The main motivator for patients to join a club was that the clubs reduced the amount of time it took patients to get their medications, something that has been shown to be a strong barrier to treatment adherence:

"Okay, like clubs, most people prefer clubs, because if they hear that so and so has joined the club, they don't waste time to get the service, they come and go immediately, they also insist that they be included in the clubs, because they don't want to wait for long hours in the queue."

Clinical Provider, Intervention site

".. Oh, it's very quick. Like, it's super, super-quick. Uh, not even five minutes. Depending on how long it takes me to find the medication... It's maybe two, three minutes or something."

Clinical Provider, Control site

Further, providers suggested that the clubs have been successful not only at decongesting the clinics and reducing the amount of time patients needed to spend at the clinic, but that they have also been helpful in reducing the rate of people defaulting from the treatment programs. This is consistent with what we have observed in the effectiveness evaluation:

"The other one is these clubs, they have made it possible for people to be able to drink the pills. In all truth, since we began using these clubs, right, the rate of people who are drinking the pills, who are defaulting has decreased." Non-Clinical Facility Staff, Intervention Site 
Providers also noted that one of the benefits of Adherence Clubs included the general notion of having a community of support for continuing to take their treatment and adhere to treatment schedules as it gave them a place to discuss issues they were having with people who had been through similar situations. They noted that patients used the clubs as a place to get ideas on how to cope with the difficulties of treatment with a sympathetic audience:

"But I think [support groups] are very much important because a person will meet others who are in the same situation as her or him. So they will discuss something that they go through together and with same situations. So they will be able to discuss those same situations together. They know that they are HIV positive ..., they will be able to give each other ideas about how to cope with the disease, how to cope with stress, how to take the medication. So they are very much important. If they are together, they will be able to share ideas."

Clinical Provider, Control site

Providers also noted that participants appreciated that being in a club allowed them to have someone else access their medication for them if they could not attend the clinic, something we also saw was a common theme in our patient focus group discussions.

"Yes, even the support group. They say there's a club, it's a support club, it can also help because one person may come and collect and then they can give the patient, the medication to the patient and they can also advise each other on that club."

Clinical Provider, Control site

Despite the positive aspects noted of adherence clubs, they also frequently attracted negative views by providers. Additional weaknesses of Adherence clubs included that providers perceived that some patients preferred to just come in and take their medication and leave rather than staying for an hour long for club activities. In other words, some patients were perceived as using the clubs much more like a DMD pickup point than AC:

"There are now few people who want to come to clubs because we should run a club for an hour because in the club we have to educate them and do everything, measure them and do everything. So, you find that they are not interested here because they come and take their medication and go. So that's that."

Non-Clinical Facility Staff, Intervention Site

This may be because, as was noted in the patient focus groups and interviews, clubs allowed patients to get longer scripts and make fewer trips to receive their medications. Still, it is also important to note that this seems to be a misconception about the requirements for Adherence Clubs as the AGL does not state that patient must stay for additional education. It is not clear whether this is a misconception by patients or providers, but given this report focuses on the provider perspective this may be on the provider side and this may need to be made clearer whilst training staff to implement these interventions. 
While they appeared popular with patients and many providers, this was not universally true. In some cases providers noted that adherence clubs placed a burden on staff and staffing shortages limited the effectiveness of clubs as there were not enough nurses to attend every adherence club meeting. Some respondents also felt that adherence clubs were not effective and were phased out in favour of DMD/CCMDD (as we know happened at some of the intervention facilities).

"We did try to open the support group, [inaudible] adherence club, but really, they don't work. It was not easy. Because, like I said, since... Starting from 2013, or 2014, we have started it. Before that, we tried to start it, but it didn't work. So, after getting two people, three people, they just recruit them. It was not easy. Most of the people they have to look after their children. Only, the thing that they, the idea of coming to the clinic for their meeting, if they don't come for their medication, it's only the time that they cannot do that. That's why some of the things delayed." Non-Clinical Facility Staff, Control site

It is unclear how much this transition to DMD over AC was related to the push to decongest clinics (which occurred during the follow up period), or if clubs were genuinely seen, at some sites, as too difficult or not effective. In some instances, it was also not clear whether clubs were being provided to the right patient, as some suggested they should be used for unstable patients, while using DMD for stable patients.

"We do have, we used to have I'd say because in a sense we dismantled in a favour of CCMDD which is a Chronic Medication Distribution and Dispensation. We used to have the adherence ART clubs where patients would come in groups, have had discussions. We would give him the education, we would also give him talks aimed at improving and enhancing their adherence to medication. But recently in favour of CCMDD we have dismantled those clubs. But still patients always will want to come in groups to have, they have talks and discussions pertaining to adherence to medication. But besides that only the clients that are compliant and patients that are adherent properly to their treatment are recruited for CCMDD. With other clients that are unstable or need regular attention we still keep them in clubs. So we do have clubs, adherence clubs but at the same time we have adopted so much the concept of CCMDD." Clinical Provider, Control Site - DMD Control*

Some providers also expressed that initially patients were sceptical about the motivations of clinic staff for moving them to clubs and had concern that they would be given worse care and this was a barrier to getting patients to enrol in clubs (and also DMD): 
"Because the clubs has been there for a long time. With the CCMDD, they were quite reluctant, because they were... They thought maybe we're just trying to chase them away from the facility. Others they just get the satisfaction if they come to the facility. But we tried, and tried, until they get used to it. Though others, they still... They say no I don't want. Even the patient is stable, they don't want to go to be recruited to CCMDD. Though it's a small number, but initially it was a challenge, because they had this in mind that, no these people, they don't want us here, they're chasing us out. [Laughs]. Even today, to others, we're still trying. But it's happening anyway."

Clinical Provider, Intervention site - DMD intervention at enrollment

\section{Decentralized Medicine Delivery}

The main benefit that providers identified around DMD was that it was a useful intervention for alleviating the crowds and the long queues at the clinics, consistent with the push to decongest clinics through use of DMD. This was true for both models of DMD, CCMDD and CDU.

"They help us a lot because a number of people that were coming to the facilities that went to the CCMDD's, they reduced the queue, low queues of the facilities, of the facility and the adherence, we've no longer have, they shan't waiting for their medication for so long because they just come to the pick-up point and pick their medication and go home." Non-Clinical Facility Staff, Intervention site - DMD at enrollment

"And then with CCMDD, let me tell you, with CCMDD, it was a good thing because patients, we used to have a shift of patients from our facility. Patients were mostly complaining of waiting time but because we introduced that and we explained to them that it's going to assist them to reduce their waiting time at the facility and then to reduce the number of them coming to the facility. Hence, they agreed and they were satisfied about it and we could see the difference after introduction of that." Clinical Provider, Control site

Respondents additionally noted that DMD was useful for patients, particularly those who are poor as it is easier, more affordable, and the patients do not have to queue to receive their medications:

"It's easier for patients to collect their medication, so they don't have to get, every time they... sit in the queue every time they need their medications, only every six months when they need a new prescription. So it's much easier for them, and I feel like that encourages adherence as well, because they're spending less time in the... sitting in queues. They feel like it's... they feel like they're not wasting their time as much. So they're only doing that every six months. I feel like... yes, I feel like it encourages adherence as well." Clinical provider, Control site - DMD at enrollment 
Providers noted that the benefits of DMD were not only to the patients who directly received the service but also to the clinic population in general because overall queues at the clinic were shorter. Thus providers liked it because they felt they were able to provide better care to more complicated patients. This is something we also noted in patient focus group discussions:

"CCMDD, I'll say CCMDD is good because the patients are not queueing. It makes the queue not to be long and then at the end of the day, we will be able to give quality care. If there are a lot of patients, it's difficult to give quality care. You can give it but it's difficult because there are many. So ... CCMDD, the queues will be minimised. There won't be a long queue. For those who are sick maybe, it won't be a long queue. And then for CCMDD, they just go straight to the pharmacy. They just go straight to Clicks. So it's very much important, CCMDD."

Clinical Provider, Control site - DMD at enrollment

Still other providers noted that in addition to reducing the time it takes to access medication, DMD was a strategy that patients could use to avoid stigma:

"The advantage, it saves time. It also helps with their... like, there are those who are afraid to be seen at the clinic, so it helps them in the other way, because they go straight there going ... to the to the reception, you know? So that's the advantage for those who are afraid to come to the facility, think of stigma, all the stuff. Yes, it minimises the stigma thing." Outreach Staff, Control site - DMD at enrollment

While some intervention respondents said there was no difficulty in implementing DMD, some implementation challenges did emerge particularly with the CCMDD model. Because some patients were now collecting their medicine outside the clinic, they had to implement new processes for these patients when they did return to the facility because they were not able to find their medication at the CCMDD pickup point. This was a common theme among patients as well:

"It's just that when there are two lines...remember people who are coming for CCMDD/DMD they do not have to wait. So these people they didn't find their medication there by the hall I am making an example by the hall then they come back to the facility. When they come back to the facility there is a line for the facility and there is also a line for those people who won't wait because we told them that in CCMDD you do not wait, remember. So you find that the sister who is working on chronic days is having a problem because she has to deal with these two lines at the same time." 
In addition, providers found that completing documentation of patients in DMD (as well as AC) was challenging, as illustrated below:

"The reason I'm saying that is because of we still have challenges in both programmes. The CCMDD we still have a challenge of the capturing of the enrollment forms and then on the adherence club we still have the challenges of professional nurse who would be that, who would be attending the adherence day, every time they have been here." Non-Clinical Facility Staff, Intervention site - DMD at enrollment

Some providers suggested that additional personnel were needed to be able to deal with the additional burden this created, and that it was now more difficult to keep track of patients enrolled in DMD because they do not have a dedicated person capturing information from DMD sites:

“CCMDD, it's a challenge now. When we started in February last year, it was going smoothly but now recently, we've got a problem with CCMDD. The thing is that patients are no longer... Let me say we, what can I say? We enrol patients but their enrollment forms are no longer captured. Remember..., we started in February and all those patients that were started in February are supposed to be re-enrolled again. But now the challenge is that we don't have a site management or site person who is capturing our enrollment forms. We've got lots and lots of forms that are not enrolled."

Non-Clinical Facility Staff, Control site - DMD at enrollment

Still others noted that stationary shortages and other resource limitations made enrolling patients onto DMD and documenting their care on DMD difficult:

"They play a very big role, the resources. Like, if you don't have the forms, you won't be able to enrol the patient because... I never saw the original paper on CCMDD, I only saw the copies. So sometimes there's no, the copy machine isn't working because there's no ink, maybe for the whole week, no ink, no ink. So you won't have that enrollment form. So you won't be able to enrol. So the resources are, they can hinder professional intervention."

Clinical provider, Control site - DMD at enrollment

A common weakness of CCMDD/DMD identified by both intervention and control sites, included what they referred to as "stock-outs", where patients did not find medication at their pick-up point collection site and therefore had to return to the clinic: 
"To be honest with you, they're not delivering on time. They're delivering late, so sometimes the patients are coming to collect and then their medicines haven't even been delivered yet. And then, the medications will only be delivered, like, a week or a couple of days after the date that the patients were supposed to come and collect. So that's basically been the biggest problem. Another problem is a lot of the patients are not collecting. That's also a major... So, we have a lot of packages that are just not being collected. But yes, I would say that's about the biggest disadvantage."

Clinical Provider, Control site - DMD at enrollment

It is unclear whether the shortages are true stock outs, where there is no drug, or, more likely whether the medication is simply not at the pickup point at the time it is needed as illustrated by the quotes below:

“...when it's good, it's good; but then, we don't receive stock and they actually don't send the packages, and then it's bad."

Clinical Provider, Control site - DMD at enrollment

Providers specifically noted that the issue around drugs was related to CCMDD and not to some of the other strategies because of the way that the programs are managed:

"But these ones in the clubs and fast lane because their pills come from the hospital, but the ones in the CCMDD queue, it's problematic. Sometimes you find that they did not bring them. Other times they brought only one packet. So even the clients begin to get unhappy."

Non-Clinical Facility Staff, Intervention site - DMD at enrollment

Still others noted that when DMD failed to work well that it created additional burdens on providers that they would not otherwise have:

"Okay, disadvantages, though, a special, like I mentioned before, those who can't find their medication, are still a challenge. And they come back to us, because if we come in the clinic, we still have to attend them. We cannot make them join the queue, if they are here now, you have to attend them first before those who are in the queue."

Non-Clinical Facility Staff, Intervention site - DMD at enrollment

It was commonly noted that in cases where DMD did not go as planned, it affected the impressions that patients had of the quality of care they were receiving and that word of the drugs not being at the pickup points spread quickly and reduced patients' willingness to enrol in DMD: 
"Serious. They are complaining and if you recruiting a patient and tell them about CCMDD they would say: no we are not interested we heard that if you go to a point we are not gonna get our medication - so I walk a long, what-d'you-call-it, road coming to the clinic after I went to doctor so-and-so and didn't get my medication. So I won't lie to you they don't like CCMDD."

Non-Clinical Facility Staff, Intervention site - DMD at enrollment

Still other providers noted that it affected their own morale when patients arrived at a DMD pick up point and could not receive their medications and that this failing had a cost to patients in terms of transport and time away from their daily activities:

"It hurts when a person knocks here. I open for them and check their cards and realize that they have arrived to collect on the exact date stated on their card and yet they did not receive their parcel. They complain about transport fare and unfortunately I also cannot help them with that. I do not have the money."

Clinical provider, Control site - DMD Control*

Lastly, respondents indicated that patients appeared to be confused around the prescriptions and the pick-up points, which at times actually caused more congestion in the clinic:

"And also, they don't understand that that CCMDD... it's... they are given a script and a script is only valid for so long, depending on how long the script is written up for. So it can be four months, they're working on a four-month or a six-month system... And that's also another problem. The patients are coming to collect the medication, but then their script is up, so then they actually need to get their file and go through the system with the sisters again, to put them back into the system." Clinical Provider, Control site

"Maybe they will go once and maybe they were supposed to go maybe two months, they will only go once and then they come back for another prescription, you see. They said, I must come back to re-prescribe. So that's the disadvantages because the patient who goes only once a month and we expected that patient maybe to go for three months or two months. So the patient will go only for one month and then come back for re-prescription"

Clinical provider, control site

\subsubsection{Discussion: Patients stable and adherent}

A number of contextual factors should be noted as we interpret the results of the interviews with providers. First, only one site in our sample was a pure control site, not yet implementing any form of DMD (Limpopo, Table 1.1). All other sites that were allocated to the control group in the impact evaluation were either implementing DMD or had started implementing DMD after the HIV cohort enrolment - this was the case for both CCMDD in North West, Limpopo and KwaZulu Natal and for CDU in Ekurhuleni. Therefore, it is not surprising that we see strong themes emerging in both intervention and control groups around DMD. 
Second, it should be noted that shortly after AGL implementation started at the early learning sites the NDOH prioritised a 'decongestion strategy' to decant stable patients out of the facility. This strategy prioritized the repeat prescription collection strategies (AC, DMD and spaced fast lane appointments) from the AGL and set high targets for the number of patients to be transitioned to DMD. As a result, several facilities that previously had been implementing adherence clubs took whole cohorts of patients off $\mathrm{AC}$ and put them onto DMD-this was seen in all three districts implementing CCMDD. It is not clear if patients were given a choice and it has caused confusion.

Still, even with these difficulties, repeat prescription strategies like DMD were seen as positive and that when they worked well, were generally seen as desirable for patients. They shortened the time that it took patients to get medications and reduced the number of patients at the clinics allowing providers to focus on patients who were not stable. Providers did note issues with RPCS, particularly DMD as it created difficulties for patients and providers when it was poorly implemented or drugs were not available when patients

...repeat prescription strategies like DMD were seen as positive and that when they worked well, were generally seen as desirable for patients. presented for their medications or patients allowed their prescriptions to expire and thus had to return to the clinic for another prescription. Additionally, while it did not emerge directly from the IDI respondents nor did we ask directly, the providers alluded to frustration about not knowing exactly what was happening with their patients at pick-up points. This may suggest a challenge with data flowing from the pick-up points back to the clinics.

\subsubsection{Key Messages: Patients stable and adherent}

i. Stable patient had several options for enrolling into a repeat prescription strategy and providers saw this as being a positive addition to the clinics

ii. Adherence clubs were generally viewed favourably by providers as they allowed patients to meet in groups and provide support to each other with a potential increase in adherence

iii. DMD was also seen as a good way to decongest clinics, though it was noted that when it was poorly implemented and patients could not receive their medications when scheduled to do so, it created problems for patients and providers

\subsubsection{Results: Patients failing treatment or who missed visits}

AGL interventions for patients who are unstable on treatment (either failing treatment or nonadherent) or at risk of being lost from care focus primarily on retention strategies: Enhanced Adherence Counseling (EAC) and Tracing and retention in care (TRIC). 


\section{Enhanced Adherence Counseling (EAC)}

Intervention respondents perceived individual counselling for patients with poor adherence as important because it helps patients with an elevated viral load knowing how to take their medication, when to take their medication, and how to recognize side effects to expect and report. A respondent illustrates this below:

"It is popular, because everyone who has to do enhanced adherence counselling. If you come for visits, you make sure that you do enhanced adherence counselling. Especially if you have a high viral load."

Non-Clinical Facility Staff, Intervention site

However providers noted that while they saw EAC as important, that it was resource intensive and was not always possible to implement if the providers were not available to do so:

"Maybe there's a drop, or let me say, there... Maybe there's a problem with the viral load. It's on and off, it's not stable. So we just send the patient, or we do a little bit of it as I'm saying.

Because we may find that in the whole facility, there's only one sister. So you can't sometimes do all those things together. So if there's a lay counsellor available, then we send the patient. So that the lay counsellor will pick up the problems that the patient... Maybe he's got a viral load that is not supressed, or the viral load that is on and off, maybe due to other problems that we are unable to pick up here. So that's how we do."

Clinical Provider, Intervention Site

Providers from the control sites also explained that they make extra counselling efforts in an attempt to retain people in care, even calling their efforts by the AGL name, "enhanced adherence counselling." Providers at control sites frequently expressed using viral load to monitor patients.

"Those who are missing their doses and those... we do adherence counselling to those whom their viral load is not suppressing. After one we check the viral load we find out that their viral load is not suppressing and some of the patient, even the family members, they do come here and tell us that they don't take treatment, so we also do adherence counselling to those and those who we trace, those who are used to follow up."

Clinical Provider, Control site

"But if the viral load is above 1,000 then we do adherence counselling and enhanced adherence counselling. Because visually we always [pause] the high viral load can be attributed to poor adherence. So, if we trust that we hope that the patient will be suppressed after addressing the issues of adherence."

Clinical Provider, Control site 


\section{Tracing and Retention in Care (TRIC)}

Similar themes emerged around the strengths and weaknesses of tracing patients in both the intervention and control groups. One important strength of tracing identified by intervention providers was that it not only helped them find patients that are sick because they have not been taking medication, but they also found that those patients were appreciative of the efforts made by the staff and often then returned to the clinic. One respondent illustrates this theme:

"Yes, they are changing, especially with tracing because sometimes we find out that other patients, they will appreciate that you come in the house, you trace them and they go back to the clinic. Because other patient you will find out they are being scared to come back with their... because they missed their dates. But us talking to them in the community, it helped them

$$
\text { a lot" }
$$

Outreach Staff, Intervention site

We note that fear of coming back to the clinic after defaulting or missing a visit was noted by the providers, though not by patients in our interviews. It is also worth noting that these findings are somewhat in contrast with the effectiveness intervention. There we did not find a benefit to tracing overall, though we did find that tracing was targeted towards those most likely to come back.

Tracing, while not implemented to the same intensity as at the intervention sites, is supposed to be performed at all clinics, even control sites. Providers from some control sites discussed their partnerships with local Non-Governmental Organizations (NGO) to ensure follow up and retention in care:

"Because we have the Aurum people who make sure that this patient comes to the clinic. They call them, they call them and remind them, don't forget your date when you are coming to take bloods, this date, don't forget that. And also, we have the Aurum people who are initiating them and then the counsellors who are doing the adherence, like the one from COBALT who is doing the adherence. So we make sure by them, those people, they really make sure."

Clinical provider, Control site

Intervention site respondents most frequently said the challenges of implementing missed visit tracking included a lack of adequate resources, specifically transport, cell phones, or human resources, as well as general safety concerns. These key themes are illustrated by intervention respondents below: 
"Okay. When we going out we've got the challenges that sometimes you find out when you go there that the gate is locked and there is a dogs. They normally like to open that gate so that they would be bite by the dogs and most of the people they are at work so we can't register them. And there is no transport when you going outside. Sometimes we'll find that, you must go in a far distance to do the registration. And there is more NGO's in our area now. We've got four of them, so we are working a same work and most of the time they used to say they coming from the facility, while they not coming from [site]..."

Outreach Staff, Intervention site

Lack of phones or airtime, or an inability to access phones as only the facility management had the PIN code was a common barrier to implementing tracing, and one that our team observed quite frequently when we went to the sites:

"With tracing, we do trace our patient, but there is a challenge in such a way that we are having one telephone system that we're using. In our telephones, the challenge that we having is that we are two professional [nurses] that we using the pin code."

Clinical Provider, Intervention site

Providers also noted that there were too many patients who needed to be traced for the community outreach workers to trace all of them:

"And we do also have the CCGs. Our Community Care Givers. We engage them a lot by tracing these patients. And though we don't have a whole number of patients... I mean of CCGs to trace our patients. We still need more. Since, when I'm talking about this facility. This facility now, is having a population of more than 22,000 people who comes into this facility. So, by having a scanty number of CCGs, so sometimes we get in difficult where there are no-go areas where they can't reach the other areas. But with the help of mobiles, and... They are able. But those are the missing's that we are still having. In such a way that we have a high number of those loss to follow patients. Those are the challenges."

Clinical Provider. intervention site

Several providers noted that there was some disconnect between the clinics and the staff who did the tracing. One provider also noted a common problem with tracing, that patients frequently change their phone numbers/sim cards and therefore the numbers they gave to be traced do not work: 
"Unfortunately in this facility, we don't have the data capturer, we are assisted by the data capturer from the NGO MSF that is supporting us around here. So should we ever we want to see those patients that we need to trace, we go through her. And she's going to give us the list. Then we've got the phone, then we ask from the... Our operational manager, we try to contact those patients or their relatives. Though the challenge is that they change the sim cards now and then.

So if we fail in that way, we'll... We try to utilise the CCGs to trace the patient."

Clinical Provider, Intervention site

Additionally, intervention respondents articulated that challenges included patients providing an incorrect address or moving on frequently from the address that was given making it difficult to trace them.

"Some of them they are just writing the wrong addresses, the wrong street numbers so that is hard to trace them. But when we go, some of them they are the tenants neh? They don't stay long, they just move out. So when we go to those houses, we ask the landlord, where is she? If she's in at [unclear] then we can make a report to our sisters, tell them that this people they have moved, they are no more staying at that address. They said they moved two weeks ago."

Outreach Staff, Intervention site

Similar challenges were noted among control respondents, as they suggested patients deliberately give false information:

"You come into their house, you're telling them about one, two, three, four, five, then others, they lie because they sometimes give false information to the facility. Wrong addresses and wrong names. So those kinds of things are some of the things [door squeaks] which make it difficult, you know?"

Outreach Staff, Control site

Finally providers noted difficulties in tracing patients during clinic or office hours that limited their ability to reach patients at home. Further they noted that while in some cases patients appreciated being traced, in others families and patient were not welcoming of tracers:

"Because we also have issue of communication. Sometimes we don't or we use our cell phones in tracing the patients because here at the facility we don't have a phone which we can call their numbers so we must use during office hours. So it means after work when the office is closed too we are not able to access the phone to call the patient. And even tracing of patients, some home based carers they found that in some families they are not welcome."

Clinical provider, Control site

\subsubsection{Discussion: Patients failing treatment or who missed visits}

Interpreting the results of interventions for patient who are unstable or have missed visits is complicated by the fact that most clinics do some counselling for patients who have an elevated 
viral load and do some tracing of patients who have missed visits or who are lost to follow up. These two interventions intensify the approaches by increasing the counselling and by decreasing the amount of time patients are out of care before they are traced. Still, because control sites do implement version of these interventions, it is not surprising that both intervention and control sites experienced similar issues.

\subsubsection{Key Messages: Patients failing treatment or who missed visits}

i. Providers saw enhanced adherence counselling as an important strategy for improving adherence for those with an elevated viral load. However, they noted that EAC brought an additional burden to the clinic in terms of provider time and could not always be delivered if not enough staff were available

ii. Providers also saw tracing as an important strategy for getting patients to return to care, but there was a strong feeling that they had limited resources to be able to complete tracing activities and were not always welcomed in the community to trace patients

\subsection{FINDINGS FOR EVALUATION QUESTION 3 AND 4}

Question 3: How could implementation of adherence interventions and the minimum package of interventions be improved and what additional strategies could improve adherence? and

Question 4: What additional strategies do providers feel would be helpful in improving treatment adherence?

\subsubsection{Results: Recommendations to improve implementation}

As providers discussed these two questions together, we report their results together. As seen in Table 3.7, the most frequently mentioned cross-cutting suggestion for improvements across both intervention and control respondents was similar across groups. Primarily, both groups suggested provision of adequate resources to implement interventions.

Table 3.7 Most frequently cited cross-cutting suggestions for adherence strategies among provider IDI respondents by intervention and control groups

\begin{tabular}{lll}
\hline & INTERVENTION & CONTROL \\
& $\mathrm{N}=10$ & $\mathrm{~N}=12$ \\
\hline $\begin{array}{l}\text { Cross-cutting suggestions to } \\
\text { improve implementation }\end{array}$ & - HR and Other Resources & - Resources \\
adherence strategies & - Training & - Training \\
& - Ettitude & - Attitude \\
& - Efficiency & - Efficiency and Supervision
\end{tabular}

Source: Authors 
The most frequently mentioned theme was human resources, as intervention respondents perceived a shortage of nurses and a shortage of male staff for safety. One respondent illustrates the challenge below:

"[Laughs] To be honest with you, the attitude was not that good. Because you know, the programs, they do come left and right, but they don't look up to what resources we have. Especially the human resources, so we are overworked, I can say. Yes, that's the thing. Though you can like to implement any intervention 100\%, but you can't, because we are overworked, that's what I can say. The interventions are there, they are good, mostly there are benefits to the... To our clients and community. But to us sometimes, they are not. Because we have to do a lot of things while we are only short staff."

Clinical provider. Intervention site

In particular, providers felt that they needed to increase the number of nurses at the sites as nurses were responsible for implementing many of the AGL interventions:

"There's nothing much, except that if, really, our government can increase our, I mean, nurses. That could be a really appreciated. Because, sometimes, you only to find that the nurses are working under pressure. And some of the implementation, the [inaudible] implementations, they are very resistant to do it, or to implement it. Because of they're tired, and, you know, it's too much for them. Maybe, if our government can think for us. Like, for instance, here, with me here. I'm a 24-hour service clinic, only operating with eight professional nurses. Others are on night shift, and out of those, they are working day shift. So, it's very difficult. With the huge number of those 22000 and above patients. Eight professional nurses. So, that is why our nurses, you see, it seems as if, even though they do get the information they're not able to implement it. Because they are tired. Yes."

Non-Clinical Facility Staff, Intervention site

Intervention respondents said more counsellors and health educators would be beneficial as well as additional resources such as computers to enable staff to work more efficiently:

"We do need to have more staff members we do need more support from our supporting partners. We do need to have some... We need to have some other than the materials that we have we do need to have something that is innovative maybe digital and advanced in terms of technology. To be able to see to it that the programmes are implemented properly. And they are observed and monitored on regular basis. We do have computers but they haven't installed them. I think as soon as we... They are installed I think they will help us to facilitate and to enhance the way we implement our problems."

Non-Clinical Facility Staff, Control site 
The second most common resource discussed by both groups was general supplies, including education materials, transport, masks, gloves, registers, or scales. As one respondent explains,

"They play a very big role, the resources. Like, if you don't have the forms, you won't be able to enrol the patient because... I never saw the original paper on CCMDD, I only saw the copies. So sometimes there's no, the copy machine isn't working because there's no ink, maybe for the whole week, no ink, no ink. So you won't have that enrollment form. So you won't be able to enrol. So the resources are, they can hinder professional intervention."

Clinical provider, Control site

Additional cross-cutting suggestions for improvement from both intervention and control sites was additional training and provision of supplies including education material to enable providers to better do their jobs. Intervention respondents frequently discussed training for all staff members for redundancy, in-service training, and additional trainings on the recent changes in guidelines. Control respondents most frequently discussed in-service training and HIV training for nurses. Themes that emerged around training are illustrated below:

"Reason is that I would wish that everyone in the facility must be trained so that it will be easier for everyone to be aware about what-d'you-call-it policies and their implementation - it shouldn't be that other people learn only from others because sometimes it's hard to listen to other person at the side, but if one has gone to the training it's easier to implement things immediately."

Non-Clinical Facility Staff, Intervention site

"To start with training. If every intervention, intervention training should be done. Training should be amongst the staff members. Training should be amongst the patient. And then we should also have the material, the pamphlets whereby the patient will read and it should be in all the languages, not only in English. And then also, we should have a person specifically allocated in all the facilities. Let me say, let me say, if there's adherence or the intervention is introduced, there should be specifically a person for that." Non-Clinical Facility Staff, Control site

More specific themes that emerged to improve adherence strategies for new patients, stable patients or unstable patients can be seen in Table 3.8. Additionally, in general, the control respondents offered more suggestions for improvement than the intervention group and interestingly often offered ideas that are in fact included and catered for in the AGL such as additional health education materials on healthy living and the importance of adherence (as found in the AGL flipcharts and pamphlets), and allowing other patients to pick up medications or patients to pick up their medications less frequently (as allowed in the repeat prescription collection strategies). 
Table 3.8 Most frequently cited suggestions for adherence strategies among interviewed provider by intervention and control groups

\begin{tabular}{lll}
\hline & INTERVENTION & CONTROL \\
TYPE OF PATIENT & $\mathrm{N}=10$ & $\mathrm{~N}=12$ \\
\hline & Suggestions & Suggestions \\
\hline $\begin{array}{l}\text { New patients testing and initiating } \\
\text { treatment }\end{array}$ & N/A no themes emerged & N/A no themes emerged \\
\hline Patients stable and adherent & - Health education sessions & - Health education \\
& - Reminders & - Allow others to pick up medication \\
\hline $\begin{array}{l}\text { Patients unstable on treatment (either } \\
\text { failing treatment or non-adherent) or at } \\
\text { risk of being lost }\end{array}$ & - Campaigns and radio station & - Campaigns and Reminders \\
\hline $\begin{array}{l}\text { Source: Authors } \\
\text { S }\end{array}$ & & - Increase home based care visits \\
\end{tabular}

As examples of the suggestions raised, the quotes below illustrate these points. In terms of home based visits, providers noted they were linked to the community and well placed to conduct adherence interventions:

"We've got community health care workers who are specifically linked with the community in specific areas in our catchment... So they are the ones who normally, when we arrange maybe a campaign, they go for... let's say, maybe we choose one of the catchment areas, then we go for what to give information to that community."

Clinical provider, Control Site

"In our facility, we have community healthcare workers who make sure that if the patient is not adhering, they go and visit those patients."

Clinical provider, Control Site

Educating the community was also noted as a way to promote adherence and treatment initiation by letting the community know what programs are available at the clinics:

"I think we have to involve the community at large, ... so that they will tell the community that there are these programs. Because normally the community will complain of everything, we are taking too long. So if at least the community members ... will just tell the community that we are just having these programs or interventions to help them, maybe it's that way."

Clinical provider, Intervention site 
Providers noted that health education has had to adapt to the new test and treat guidelines and that health campaigns need to be part of this approach, especially to get patients into care earlier:

"Normally when the patient comes in the facility for maybe checking of their status, they come and counsel and test and then we immediately start on treatment. It's not like before where we were supposed to wait for some blood results before we can start on patients. But automatically when they come, irrespective of results and everything, they are supposed to be started on treatment. We do campaigns, we health educate the community outside to come forward for testing of HIV and AIDS."

Clinical provider, Control Site

It was commonly suggested that having health education done by health promoters outside the clinic was an important approach to advocating for good adherence:

"And another thing, we encourage our health promoter to do health education whereby they go outside to the community churches. There are these community meetings that are held in Bafokeng and then they are asking to be part of that so that they can educate or give information regarding the HIV and AIDS."

Clinical provider - Control group

Providers suggested it was important to provide education to all patients, not just those on treatment.

"Yes, with the health talk, we do not do it only for the people who are taking the pills, even the ones who do not, during other times you find that they are still yet to take them, right. Yes, so now we also tell them and talk to them. When they would like to ask questions they can ask questions."

Non-Clinical Facility Staff - Intervention site

Interestingly, use of radio stations to educate about treatment and adherence was cited by the providers as ways to improve adherence and as a way to keep patients informed about the new services being offered (such as DMD) so that they did not feel they were being pushed away from the facilities:

"I think if our health promoter can do that every morning when she comes to work, teach them and tell them, if they take their medication correctly they will end up coming and collecting their medication and if there can be campaigns and support group where they can talk and if they can use, if they can report it to the radio stations, it will be much better."

Non-Clinical Facility Staff, Intervention site 
Many of the providers noted the use of educational materials as being an important part of their work in promoting adherence, this included those control sites that were implementing DMD and therefore also had access to the AGL support materials:

"I think the flyers, the pamphlets, so that the patient must know what is CCMDD and to let other people know outside, even if it's not only patients who are coming to the facility. And I wish also before it was the CCMDD introduced, they could have started with the community first to let them know that we are starting with this. Even if they will say we are the ones who were supposed to go to the community and explain to them. But maybe if they started with the patient, explaining and giving them information and then coming to us, training, sort of, training, then it was going to be better."

Clinical provider, Control Site

"I think they make it, pamphlets which is written in all different languages so that even if they went, even if they go home they'll be able to read on their own or give their children to read to them." Clinical provider, Control Site

It was also a common theme for providers to note the importance of involving community leaders in promoting treatment and adherence by promoting the work of the clinic and community outreach staff:

"I think if we can meet with the community leader explain to them or like having a meeting in the community like if we come to the community so that they will understand what are we doing..."

Non-Clinical Facility Staff, Intervention site

Finally, providers noted the use of mass media and radio as important ways to communicate about treatment and adherence and reinforce messages from the clinic:

"I think we should use mass media to communicate with our clients like radio and local newspapers in order for them to know that if you are sent to the other side it doesn't mean you are fired from the clinic. If you are tracing, we are coming to their houses, we want them back in the system. They should know the importance of them taking treatment."

Clinical provider, Intervention site

"Yeah with time some of the patients are learning to, they are accepting the changes we are having but others they still don't understand. That is why I said we need to reinforce via mass media and educate our clients then at least next time they will understand what is happening."

Clinical provider, Intervention site 


\subsubsection{Discussion: Recommendations to improve implementation}

In general, suggestions to improve implementation of the adherence interventions were broad and crosscutting, rather than specific to each of the AGL interventions. Providers generally suggested that in order to improve the interventions, more resources are needed, in particular more staff time. Both intervention and control sites perceived a need to strengthen human resource capacity by training and hiring. However, providers from intervention sites preferred additional nurses, while providers from control sites preferred additional outreach or counsellor support.

Additionally, respondents from control sites expressed feeling ill prepared or unequipped to implement the AGL well, frequently discussing a lack of training, confusion around guidelines, and little or no education materials available for patients. This in part could be because some providers at certain control sites had been made aware of the AGL, particularly if they were a site selected to implement CCMDD. There has also been a degree of national scale-up in the study districts and so providers may have been aware that other facility staff had attended trainings and did not fully understand why they had currently been excluded.

Providers from both intervention and control sites suggested how the links between facilities, health care providers and communities could be leveraged to improve patient awareness and uptake of adherence interventions. Making better use of community health workers to spread messages, involving community leaders and using health promoters outside of the facilities were all highlighted as potential improvements or adaptations to support implementation of the AGL. In addition, ongoing campaigns and use of the media, particularly radio, were put forward as key ways that patients could be kept informed about health care policy changes and new services and interventions that are now available.

\subsubsection{Key Messages: Recommendations to improve implementation}

i. Providers generally suggested that in order to improve the interventions, more resources are needed, in particular more staff time

ii. Providers expressed a need for stronger initial training as well as ongoing mentorship and training

iii. Providers recommended that all sites be fully equipped with supplies, materials and trained providers

iv. Leveraging the links between community health workers and communities and involving community leaders was seen by providers as a key way to improve uptake of interventions.

v. Providers also recommended conducting campaigns and using the media (such as radio) to notify communities about health care changes and the services available 


\section{DISCUSSION}

The overall aim of this component of the evaluation is to understand key aspects of the implementation of the AGL from the perspective of the patients and providers and, in the longer term, to explore how these components relate to the effectiveness of each minimum package intervention on patient outcomes. This report elicits themes around the providers' perspectives on implementing the AGL minimum package compared to sites that are not yet implementing the full minimum package of AGL interventions.

In terms of repeat prescription strategies, provider perspectives were mixed on the effectiveness of adherence clubs. On the one hand, they were seen as a place for patients to share adherence strategies while also strongly shortening the time they needed to spend in the clinic. On the other hand, they believed that patients wanted to collect medication and depart quickly. Providers also noted that it was difficult to staff adherence clubs with all the other activities, and some sites phased them out in favour of DMD, though this was also in the context of provider concerns that patients did not like having to stay at clubs for a long duration for education sessions, something not required as part of the AGL. Interestingly, providers noted that the repeat

Interestingly, providers noted that the repeat prescription strategies were seen as providing benefits not just to the patient enrolled in them, but also to more complicated patients as providers could spend more time with them. prescription strategies were seen as providing benefits not just to the patient enrolled in them, but also to more complicated patients as providers could spend more time with them. The repeat prescription strategies also alleviated congestion in the clinics, but the challenge of medicines not being delivered in time for DMD emerged as an issue to be addressed along with the issue of information not being fed-back from the external pick up-points to the facilities and captured. The issue of medications not being available at pickup points was of particular concern as it resulted in unexpected patients back at facilities waiting for their medicines and general dissatisfaction with this service by both patients and providers.

The individual counselling aspect of both counselling interventions (FTIC and EAC) was seen by providers as a key element in improving patient adherence, but the labour intensive nature of this counselling was perceived to be a major barrier to effective implementation especially where facilities were considered to be short-staffed. With regard to tracing and TRIC, the providers perceived that some patients do not want home based carers to come to their homes and consequently tracing is challenging because patients give incorrect addresses and phone numbers. Migrant populations further complicate the issue of tracing and in many cases it is just not possible to reach patients who have missed visits either by phone or in person.

In general, based on the providers responses, readiness for implementing the AGL minimum package interventions at control sites was low as staff at these sites have yet to be trained and have 
not yet been introduced to the AGL SOPs and supporting materials and also the perception that sites felt under-resourced to implement new interventions. Providers at both control and intervention sites expressed concern about staffing, complained of being overworked and under resourced (particularly at those sites where interventions were implemented) and of not having sufficient training. Providers generally suggested that in order to improve the interventions, more resources are needed, in particular more staff time. This is perhaps indicative that while the AGL are supposed to help decongest clinics, reduce the burden on clinic staff and improve the quality of care for those patients who remain in care at the facility, this is dependent on facilities having a full staff complement and sufficient resources to implement those interventions. Where those resources are not available the administration and implementation of the AGL interventions can place an increased burden on existing staff, particularly in the early stages while establishing the interventions and identifying patients who should receive each intervention. It is critical that training, staffing and resource issues at a site level are carefully reviewed and considered as the AGL are scaled up and rolled out nationally to ensure they achieve their aim to improve patient retention and adherence and decongest stable patients out of facilities.

It is critical that training, staffing and resource issues at a site level are carefully reviewed and considered as the AGL are scaled up and rolled out nationally to ensure they achieve their aim to improve patient retention and adherence and decongest stable patients out of facilities.

Even though AGL supporting materials were not specifically highlighted by providers in intervention facilities, the need for such tools was raised by providers at control sites suggesting that these materials are necessary and have been accepted and well received at intervention sites. However, the lack of comment on these materials by intervention providers may also indicate that these materials may need to be more readily available for all cadres of staff at facilities and access to these materials should be assessed and addressed at each facility as national AGL implementation continues.

The implementation of the AGL, Decongestion strategy and treatment for all at similar times, and competing priorities and targets associated with each of these strategies, has led to some confusion about how interventions should be implemented. The language used to describe certain interventions by providers indicates a lack of clarity and lack of understanding about the different interventions and strategies (e.g., fast tracking initiation, same day treatment initiation, and fasttracking treatment of patients already on treatment). As national rollout of the AGL continues, focus needs to be given to training all relevant staff and cadres of health care workers and ensuring their understanding of the interventions and how the AGL and decongestion strategy overlap. Providers also need clarity on prioritising the implementation of these interventions alongside the implementation of 'test and treat' and other healthcare guidelines that may be rolled out over the same period. Additional patient education and awareness campaigns and use of the media to inform patients about these changes and the new services available could also help ensure uptake of these services. 


\subsection{KEY MESSAGES AND RECOMMENDATIONS}

- The potential for the AGL to reduce the burden on clinic staff is recognised but only if there are sufficient HR resources to implement the guidelines. Otherwise implementation of these interventions is seen as an additional burden on existing staff resulting in poor implementation and potentially poor patient care. At scale-up it is important to ensure that HR staff shortages are addressed particularly for non-clinical facility cadres such as lay counsellors, data capturers and community health workers who play a key role in AGL implementation.

- The issue of readiness of sites for implementation (i.e. materials, supplies, training, etc.) needs to be addressed before scale up so that providers feel empowered to implement effectively and have the support of their district managers.

- It is important to engage with staff to identify the appropriate people for training; adequate initial training and ongoing mentorship of staff are also key to ensuring effective implementation of the minimum package interventions.

- Targets and priorities for decongestion of patients and test and treat need to be clearly communicated in the context of implementing the AGL and appropriate training for all guidelines and strategies is required.

- Issues around DMD implementation need to be resolved, adequate systems need to be put in place to ensure that relevant data are fed back to the facilities and the ability to get the medications to the pickup points at the times when patients are supposed to pick up medications must be addressed to prevent patients becoming disheartened with this intervention, risking patient adherence and potentially returning to and causing further congestion at the facilities.

- One of the key strengths of the repeat prescription collection strategies was the element of patient choice in terms of choosing whether they prefer adherence clubs, picking their medications up at a convenient pick-up point outside of the facility, or through a spaced fast lane appoint system at the facility. Providers recognised that some patients clearly prefer the opportunity to meet as a group and provide support to each other. Ceasing adherence club activities in favour of CCMDD and dictating where patients receive their treatment should be avoided. Rather the appropriate resources must be kept in place to ensure this element of patient choice remains.

- While the counselling component of FTIC and EAC was favourably received, the introduction of adherence plans and completion of these during these counselling sessions has caused confusion about how these should be completed and by whom, and they are currently perceived as an additional burden. Continued use of these plans needs to be carefully considered and, if continued, detailed training on their completion and accessing replacement books is required.

- The need for the type of supporting materials developed for the AGL was highlighted but these materials may need to be more readily available for facilities and all cadres of clinic staff as national scale up of the AGL continues. 
- Links with communities and community leaders should be leveraged to promote adherence and treatment services and media sources should be utilised to inform patients about changes to health care provision and the introduction of new services. 


\section{REFERENCES}

1. Rosen S, Fox MP. Retention in HIV care between testing and treatment in sub-Saharan Africa: a systematic review. PLoS Med. 2011;8: e1001056. doi:10.1371/journal.pmed.1001056

2. Fox MP, Rosen S. Patient retention in antiretroviral therapy programs up to three years on treatment in sub-Saharan Africa, 2007-2009: systematic review. Trop Med Int Heal. 2010;15 Suppl 1: 1-15. doi:10.1111/j.1365-3156.2010.02508.x

3. Fox MP, Rosen S. Retention of Adult Patients on Antiretroviral Therapy in Low- and MiddleIncome Countries: Systematic Review and Meta-analysis 2008-2013. J Acquir Immune Defic Syndr. 2015;69: 98-108. doi:10.1097/QAI.0000000000000553

4. Damschroder LJ, Aron DC, Keith RE, Kirsh SR, Alexander JA, Lowery JC. Fostering implementation of health services research findings into practice: a consolidated framework for advancing implementation science. Implement Sci. 2009;4: 50. doi:10.1186/1748-5908-4-50 


\section{APPENDIX 1 - PROJECT LOGIC MODEL}

\section{A. 1 ART Gudelines Evaluation}

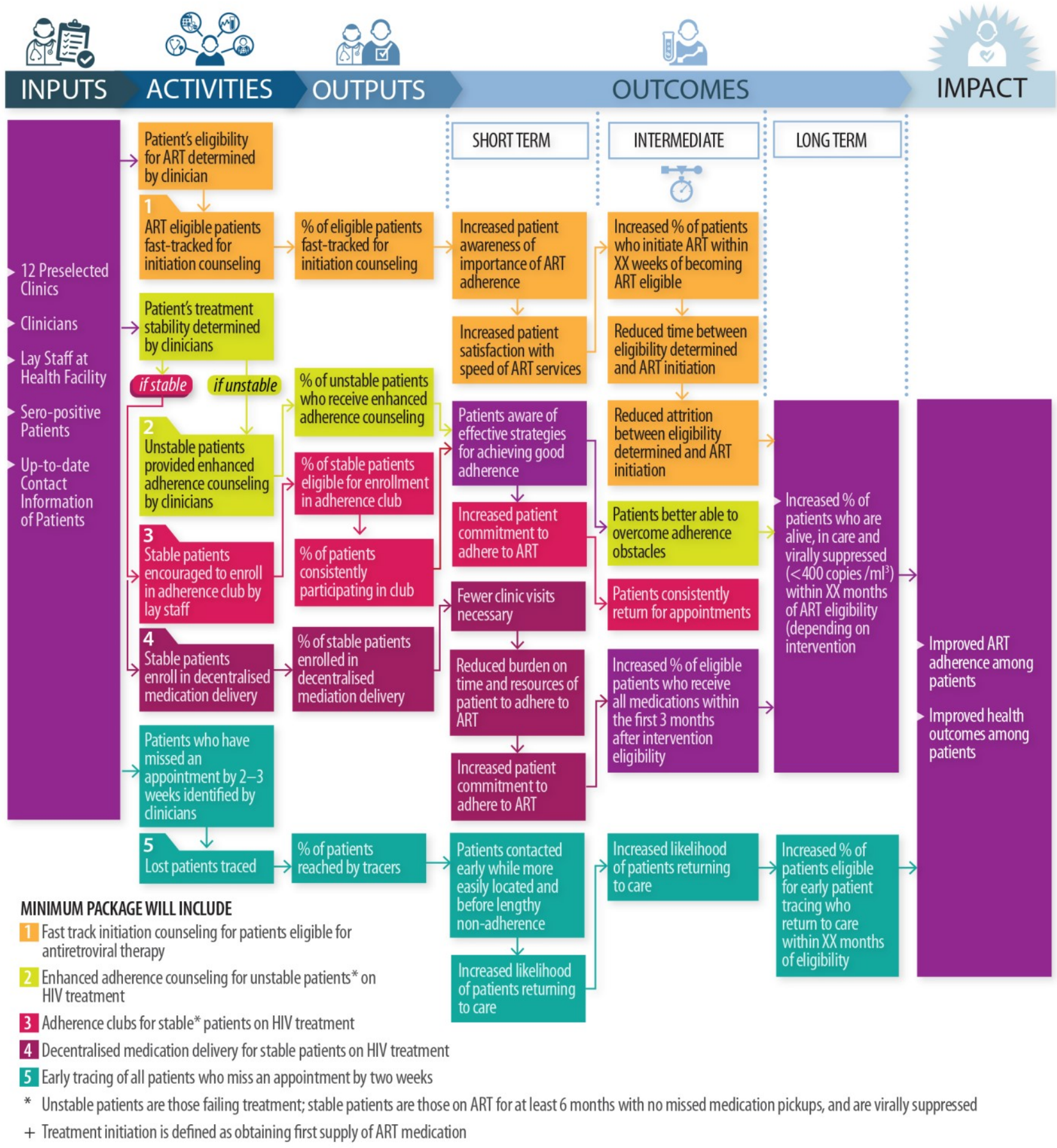

